\title{
A nodal-based implementation of a stabilized finite element method for incompressible flow problems
}

\author{
Ramon Codina*,1 \\ Universitat Politècnica de Catalunya, Jordi Girona 1-3, Edifici C1, Barcelona, Spain
}

\begin{abstract}
SUMMARY
The objective of this paper is twofold. First, a stabilized finite element method (FEM) for the incompressible Navier-Stokes is presented and several numerical experiments are conducted to check its performance. This method is capable of dealing with all the instabilities that the standard Galerkin method presents, namely the pressure instability, the instability arising in convection-dominated situations and the less popular instabilities found when the Navier-Stokes equations have a dominant Coriolis force or when there is a dominant absorption term arising from the small permeability of the medium where the flow takes place. The second objective is to describe a nodal-based implementation of the finite element formulation introduced. This implementation is based on an a priori calculation of the integrals appearing in the formulation and then the construction of the matrix and right-hand side vector of the final algebraic system to be solved. After appropriate approximations, this matrix and this vector can be constructed directly for each nodal point, without the need to loop over the elements, thus making the calculations much faster. In order to be able to do this, all the variables have to be defined at the nodes of the finite element mesh, not on the elements. This is also so for the stabilization parameters of the formulation. However, doing this gives rise to questions regarding the consistency and the conservation properties of the final scheme, which are addressed in this paper. Copyright $\mathbb{C} 2000$ John Wiley \& Sons, Ltd.
\end{abstract}

KEY WORDS: finite elements; incompressible Navier-Stokes equations; mesh graph; stabilization methods

\section{INTRODUCTION}

This paper presents a finite element formulation for solving the generalized incompressible Navier-Stokes equations, including Coriolis forces and the effect of the permeability of the medium. The formulation is based on the sub-grid scale concept, which was introduced for the scalar convection-diffusion equation in Reference [1] and generalized to systems of

\footnotetext{
* Correspondence to: ETS d'Enginyers de Camchs, CiP, Universitat Politècnica de Catalunya, Jordi Girona 1-3, Edifici C1, 08034 Barcelona, Spain.

${ }^{1}$ E-mail: codina@cimne.upc.es
} 
convection-diffusion-reaction equations in Reference [2]. This formulation applied to the generalized Navier-Stokes equations is also presented in Reference [3], where the linearized problem is analysed. The numerical analysis undertaken in this reference shows that the method is optimally convergent and that it is able to deal with the instability problems of the standard Galerkin approach. In this paper some standard benchmark problems are presented using this formulation in situations outside the scope of the classical analysis, such as thermally coupled flows or flow of non-linear materials. Likewise, the method is extended to transient problems.

The numerical instabilities of the Galerkin method that the present formulation circumvents come from very different sources. The first one is the classical inf-sup condition for the velocity-pressure finite element interpolations, which is needed in order to have pressure stability (see, e.g. Reference [4]). The second is also classical and concerns the oscillations found when the flow is dominated by convection, i.e. the cell Reynolds number is large. Finally, when the viscosity is small compared either with the Coriolis forces or with the absorption effects coming from the medium permeability, numerical oscillations may also appear (see Reference [3] for a more detailed descrintion of the instabilities arising in these cases). The stabilized formulation presented here is able to deal with all these numerical problems, allowing in particular the use of equal velocity-pressure interpolation, assumed throughout in the paper. The idea of using a stabilized method able to deal with the pressure instability and convection-dominated flows is old and is in fact the origin of the Galerkin/leastsquares method [5]. Extensions of this method to the transient incompressible Navier-Stokes equations are presented, for example, in References [6,7], among others.

Although the flexibility, generality and sound theoretical foundations of finite element methods (FEMs) applied to fluid flow have been widely acknowledged, they have been also
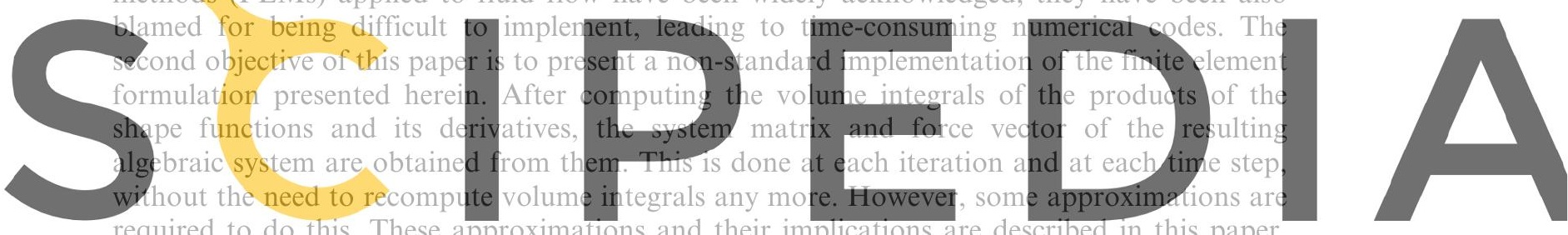

required to do this. These approximations and their implications are described in this paper.

Whereas for a standard finite element implementation, the normal flow of the calculation

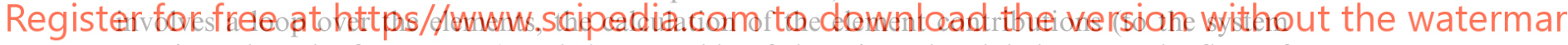
matrix and to the force vector) and the assembly of these into the global arrays, the flow of the calculations for the algorithm presented here is very different. The system matrix is formed of block matrices corresponding to the nodal points that can be obtained directly, without any reference to the elements. This is done by looping over the nodes of the finite element mesh and then over the nodes connected to a given nodal point. This involves the storage of the graph of the mesh, as well as the graph of the boundary mesh when the contributions from the boundaries need to be accounted for. It has to be stressed that, in principle, this approach is independent of the element type, and it is not restricted to linear simplicial elements as the edge-based implementations described, for example, in References [8-10].

The nodal-based implementation of the finite element formulation necessitates some approximations. First of all, all the variables need to be defined at each nodal point and the integrals where they appear have to be computed making use of their nodal values only. This is in 
particular true for the stabilization parameters of the formulation. Whereas taking them as constant over each element leads unnoticeably to the consistency and the conservativity of the formulation, these two properties might be lost when the stabilization parameters are considered to vary continuously through the interpolation of their nodal values.

The paper is organized as follows. In the following section, the general problem for a thermally coupled incompressible fluid is presented. The numerical approximation is discussed in Section 3, which includes a simple time discretization, the linearization of the equations and the finite element approximation using a stabilized formulation. Section 4 presents the nodal-based implementation of this formulation, including the approximations needed, a discussion about the consistency and conservativity of the scheme, a description of the way to store the mesh and boundary graphs and the final algorithm itself. Numerical examples are presented in Section 5. They have been chosen as representative of the approximations involved in the scheme. Results turn out to be very similar to those obtained using a standard element-based implementation, but requiring much less CPU time.

\section{PROBLEM STATEMENT}

In this section we shall consider the flow problem for an incompressible fluid in a laminat regime and taking into account several physical effects. These include the fact that the reference frame where the computational domain is attached varies in time, the coupling of the Navier-Stokes equations with the heat transport equation through the Boussinesq assumption, the permeability of the medium where the fluid flows, and a generalized Newtonian behaviour of this fluid, allowing its viscosity to depend on the invariants of the strain rate tensor.
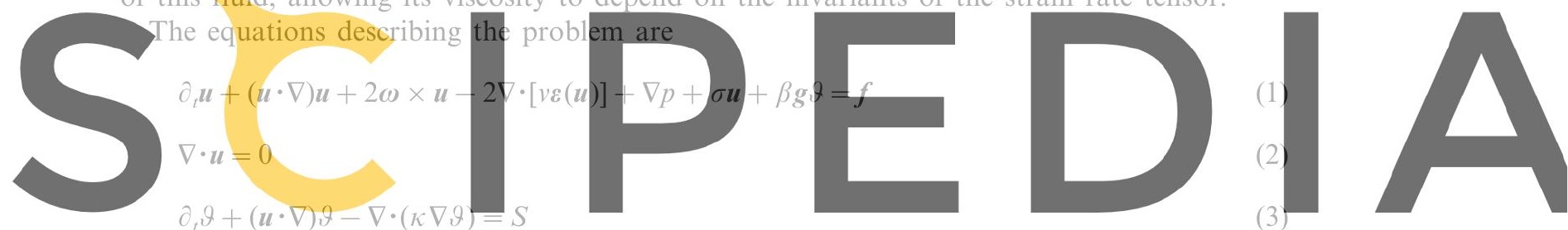

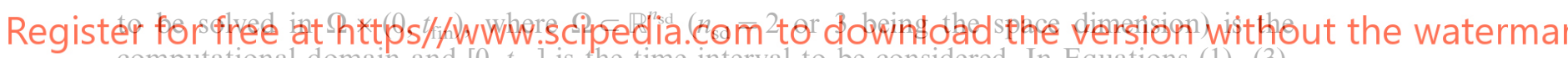
computational domaih and $\left[0, t_{\mathrm{fin}}\right]$ is the time interval to be considered. In Equations (1)-(3), $u$ denotes the velocity field, $p$ is the kinematic pressure (i.e. the pressure divided by the density), $\vartheta$ is the temperature, $v$ is the kinematic viscosity, which may depend on the invariants of the symmetrical part of the velocity gradient $\varepsilon(\boldsymbol{u}), \boldsymbol{\omega}$ is the velocity of rotation of the frame of reference (and thus $2 \omega \times \boldsymbol{u}$ is the Coriolis force), $\sigma$ is the inverse of the permeability of the medium, $\beta$ is the thermal expansion coefficient, $g$ is the gravity acceleration vector, $f$ is the vector of body forces, $\kappa$ is the thermal diffusivity (i.e. the thermal conductivity divided by the heat capacity) and $S$ is the heat source. The density $\rho_{0}$ is assumed constant to obtain Equations (1)-(3).

In the most general case, the force vector $f$ in Equation (1) contains the acceleration terms coming from the temporal variation of the reference basis and the reference buoyancy forces from the Boussinesq assumption, i.e. 


$$
\boldsymbol{f}=\boldsymbol{g}\left(1+\beta \vartheta_{0}\right)-\boldsymbol{a}_{\mathrm{fr}}-\dot{\boldsymbol{\omega}} \times \boldsymbol{x}-\boldsymbol{\omega} \times(\boldsymbol{\omega} \times \boldsymbol{x})
$$

In this equation, $\vartheta_{0}$ is the reference temperature from which buoyancy forces are computed, $\boldsymbol{a}_{\mathrm{fr}}$ is the acceleration of the frame of the reference measured from an inertial system and expressed in the moving reference and $\dot{\omega}$ is the time derivative of $\omega$. The vector of position in this moving reference has been denoted by $\boldsymbol{x}=\left(x_{1}, x_{2}, x_{3}\right) \equiv(x, y, z)$ in the three-dimensional case. Here and below, a Cartesian co-ordinate system is assumed.

The rheological behaviour that will be assumed for the fluid in one of the numerical examples is the power law, which is perhaps the most common constitutive equation for generalized Newtonian fluids. The expression of this law is

$$
v=\rho_{0}^{-1} K_{0}\left[4 I_{2}(\boldsymbol{\varepsilon})\right]^{(n-1) / 2}
$$

Here, $K_{0}$ and $n$ are physical constants $\left(K_{0}\right.$ is the material consistency and $n$ is the rate sensitivity) and $I_{2}(\varepsilon)$ is the second invariant of the strain rate tensor, $I_{2}(\varepsilon):=\varepsilon: \varepsilon / 2$, the colon standing for the double contraction of second-order tensors.

The reasons for considering such a variety of physical phenomena in the model are given now. Firstly, Coriolis forces and permeability effects have been introduced only because of generality. When they dominate, numerical oscillations may appear; global for the former, reduced to boundary layers for the latter. The stabilized method presented here allows their removal as it was shown in Reference [3].

The nodal-based implementation described later on is based on a particular way of writing the convective terms in the Navier-Stokes and the heat equation. In order to see the effect on
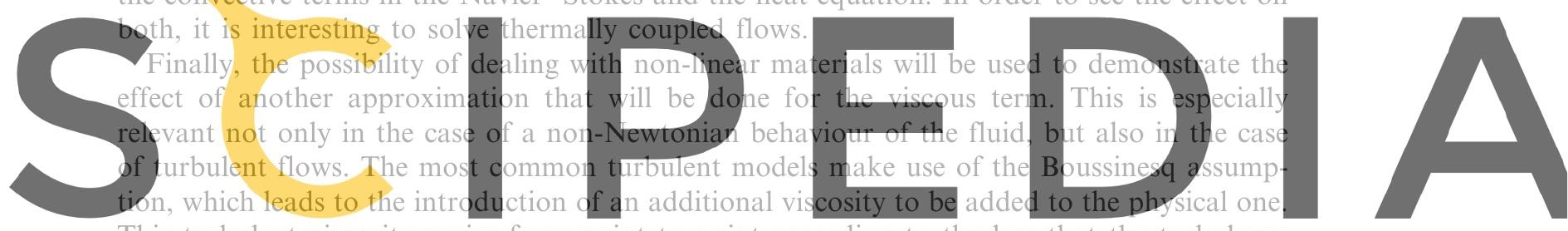

This turbulent viscosity varies from point to point according to the law that the turbulence

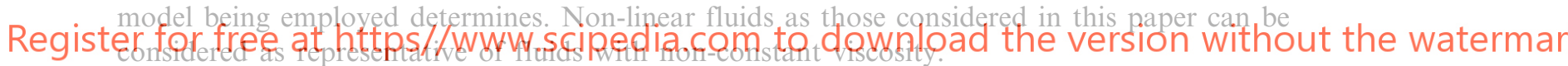

In order to write the boundary conditions for Equations (1)-(3), consider the boundary $\Gamma=\partial \Omega$ split into two sets of disjoint components as $\Gamma=\overline{\Gamma_{\mathrm{dv}} \cup \Gamma_{\mathrm{nv}}}$ and also as $\Gamma=\overline{\Gamma_{\mathrm{dt}} \cup \Gamma_{\mathrm{nt}}}$, where $\Gamma_{\mathrm{dv}}$ and $\Gamma_{\mathrm{dt}}$ are the parts of the boundary with Dirichlet type boundary conditions for the velocity and the temperature respectively, and $\Gamma_{\mathrm{nv}}$ and $\Gamma_{\mathrm{nt}}$ are those where Neumann type conditions are prescribed. If the Cauchy stress tensor (divided by the density) is written as $\boldsymbol{\sigma}=-p \boldsymbol{I}+2 \boldsymbol{v} \boldsymbol{\varepsilon}(\boldsymbol{u})$ and prescribed values are represented by an overbar, the boundary conditions to be considered are

$$
\begin{array}{lc}
\boldsymbol{u}(\boldsymbol{x}, t)=\overline{\boldsymbol{u}}(\boldsymbol{x}, t) & \text { on } \Gamma_{\mathrm{dv}} \\
\boldsymbol{n} \cdot \boldsymbol{\sigma}(\boldsymbol{x}, t)=\overline{\boldsymbol{t}}(\boldsymbol{x}, t) & \text { on } \Gamma_{\mathrm{nv}}
\end{array}
$$




$$
\begin{aligned}
& \vartheta(\boldsymbol{x}, t)=\bar{\vartheta}(\boldsymbol{x}, t) \quad \text { on } \Gamma_{\mathrm{dt}} \\
& \kappa \boldsymbol{n} \cdot \nabla \vartheta(\boldsymbol{x}, t)=\bar{h}(\boldsymbol{x}, t) \quad \text { on } \Gamma_{\mathrm{nt}}
\end{aligned}
$$

for $t \in\left(0, t_{\text {fin }}\right)$, where $\boldsymbol{n}$ is the unit normal to $\partial \Omega$.

To close the problem, initial conditions have to be appended to Equations (1)-(3) and the boundary conditions (6)-(9). They are of the form $\boldsymbol{u}(\boldsymbol{x}, 0)=\boldsymbol{u}^{0}(\boldsymbol{x}), \vartheta(\boldsymbol{x}, 0)=\vartheta^{0}(\boldsymbol{x})$ for $\boldsymbol{x} \in \Omega$, where $\boldsymbol{u}^{0}(\boldsymbol{x})$ is a given initial velocity and $\vartheta^{0}(\boldsymbol{x})$ is a given initial temperature.

\section{NUMERICAL APPROXIMATION}

\subsection{Time discrete weak form}

Let us consider now the temporal discretization of Equations (1)-(3), for which we use the generalized trapezoidal rule. Let $0=t^{0}<t^{1}<\cdots<t^{N}=t_{\text {fin }}$ be a partition to the time interval and $\alpha \in[0,1]$. To simplify the notation, we shall take the time step size $\delta t:=t^{n+1}-t^{n}$ constant for all $n$. Let us also introduce the notation
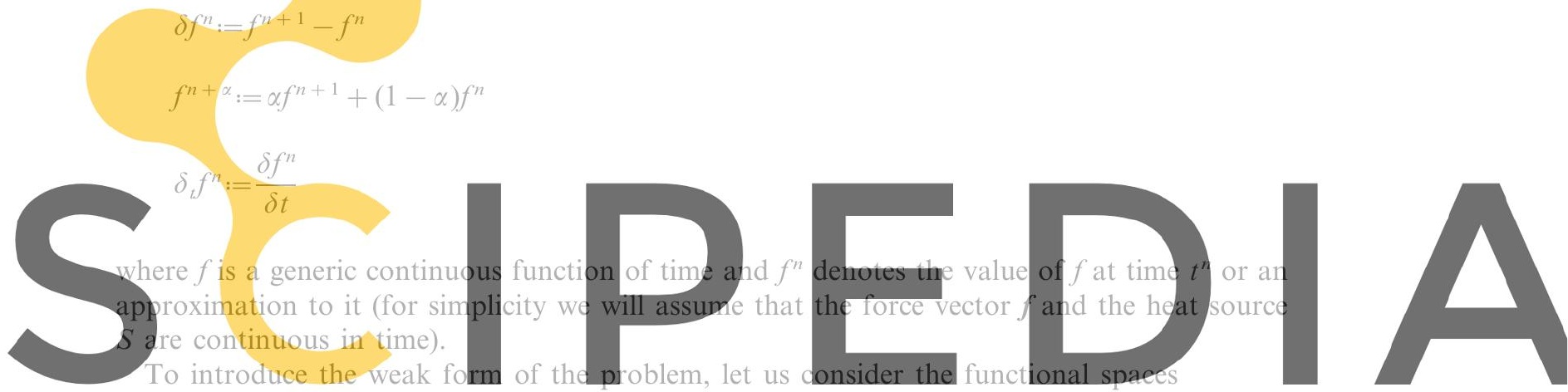

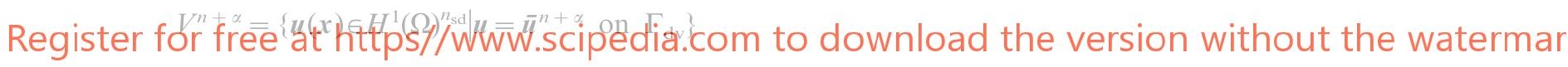

$$
\begin{aligned}
& V_{0}=\left\{u(x) \in H^{1}(\Omega)^{n_{\mathrm{sd}} \mid} u=0 \text { on } \Gamma_{\mathrm{dv}}\right\} \\
& Q=\left\{q(\boldsymbol{x}) \in L^{2}(\Omega) \mid \int_{\Omega} q \mathrm{~d} \Omega=0 \text { if } \Gamma_{\mathrm{nv}}=\varnothing\right\} \\
& \Psi^{n+\alpha}=\left\{\vartheta(\boldsymbol{x}) \in H^{1}(\Omega) \mid \vartheta=\bar{\vartheta}^{n+\alpha} \text { on } \Gamma_{\mathrm{dt}}\right\} \\
& \Psi_{0}=\left\{\vartheta(x) \in H^{1}(\Omega) \mid \vartheta=0 \text { on } \Gamma_{\mathrm{dt}}\right\}
\end{aligned}
$$

where, as usual, $L^{2}(\Omega)$ denotes the space of square integrable functions in the domain $\Omega$ and $H^{1}(\Omega)$ the sub-space of $L^{2}(\Omega)$ of functions with square integrable first derivatives. 
Once these functional spaces have been introduced, the weak form of the problem using the generalized trapezoidal rule applied to Equations (1)-(3) reads as follows: from known $\boldsymbol{u}^{n}$ and $\vartheta^{n}$, find $\boldsymbol{u}^{n+\alpha} \in V^{n+\alpha}, p^{n+1} \in Q$ and $\vartheta^{n+\alpha} \in \Psi^{n+\alpha}$ such that

$$
\begin{aligned}
& \int_{\Omega} \boldsymbol{v} \cdot\left[\delta_{t} \boldsymbol{u}^{n}+\left(\boldsymbol{u}^{n+\alpha} \cdot \nabla\right) \boldsymbol{u}^{n+\alpha}+2 \boldsymbol{\omega}^{n+\alpha} \times \boldsymbol{u}^{n+\alpha}+\sigma \boldsymbol{u}^{n+\alpha}+\boldsymbol{g} \beta \vartheta^{n+\alpha}\right] \mathrm{d} \Omega \\
& +\int_{\Omega} 2 \boldsymbol{\varepsilon}(\boldsymbol{v}): v^{n+\alpha} \boldsymbol{\varepsilon}\left(\boldsymbol{u}^{n+\alpha}\right) \mathrm{d} \Omega-\int_{\Omega} p^{n+1} \nabla \cdot \boldsymbol{v} \mathrm{d} \Omega=\int_{\Omega} \boldsymbol{v} \cdot \boldsymbol{f}^{n+\alpha} \mathrm{d} \Omega+\int_{\Gamma_{\mathrm{nv}}} \boldsymbol{v} \cdot \overline{\boldsymbol{t}}^{n+\alpha} \mathrm{d} \Gamma \\
& \int_{\Omega} q \nabla \cdot \boldsymbol{u}^{n+\alpha} \mathrm{d} \Omega=0 \\
& \int_{\Omega} \psi \cdot\left[\delta_{t} \vartheta^{n}+\left(\boldsymbol{u}^{n+\alpha} \cdot \nabla\right) \vartheta^{n+\alpha}\right] \mathrm{d} \Omega+\int_{\Omega} \kappa \nabla \psi \cdot \nabla \vartheta^{n+\alpha} \mathrm{d} \Omega=\int_{\Omega} \psi S \mathrm{~d} \Omega+\int_{\Gamma_{\mathrm{nt}}} \psi h^{n+\alpha} \mathrm{d} \Gamma
\end{aligned}
$$

for all test functions $v \in V_{0}, q \in Q$ and $\psi \in \Psi_{0}$.

The values of interest of the parameter $\alpha$ are $\alpha=\frac{1}{2}$ and $\alpha=1$, corresponding to the Crank-Nicholson and the backward Euler schemes respectively. Both are unconditionally stable, although the former is expected to be second-order accurate whereas only a first-order approximation can be expected for the latter. Under certain regularity assumptions, this is known to hold at least for the standard Galerkin method [11]; although no analysis is available for the stabilized formulation presented below (see Reference [12] for a similar approach to the transient problem for the convection-diffusion equation).
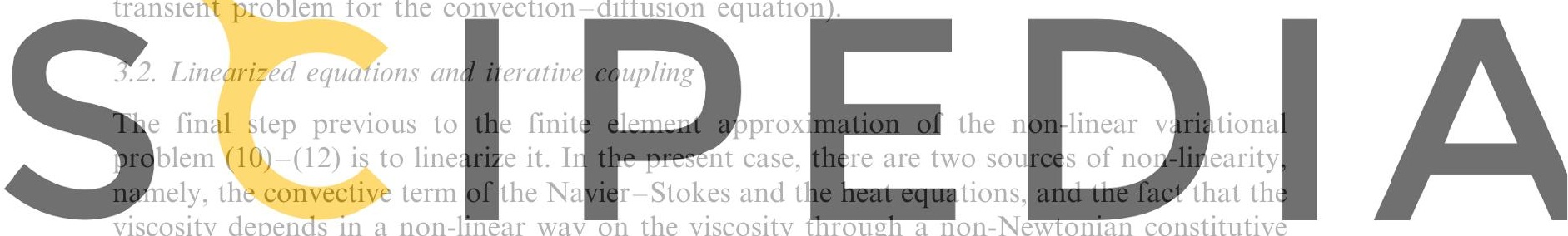
model.

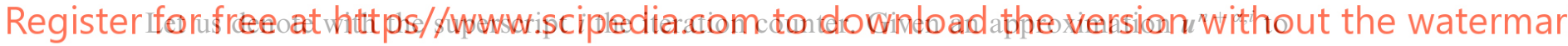

$u^{n+\alpha}$, the linearization of the convective term of the Navier-Stokes equations that we consider

is

$$
\left(\boldsymbol{u}^{n+\alpha, i+1} \cdot \nabla\right) \boldsymbol{u}^{n+\alpha, i+1} \approx\left(\boldsymbol{u}^{n+\alpha, i} \cdot \nabla\right) \boldsymbol{u}^{n+\alpha, i+1}+\lambda_{1}\left[\left(\boldsymbol{u}^{n+\alpha, i+1} \cdot \nabla\right) \boldsymbol{u}^{n+\alpha, i}-\left(\boldsymbol{u}^{n+\alpha, i} \cdot \nabla\right) \boldsymbol{u}^{n+\alpha, i}\right]
$$

where $\lambda_{1}=0$ corresponds to the Picard method and $\lambda_{1}=1$ corresponds to the Newton-Raphson scheme.

The linearization of the constitutive model (5) is not as easy as that of the convective term. In fact, the expression of the viscosity in terms of the velocity in this case is not even differentiable. In such a situation, the simplest way to deal with the constitutive non-linearity is to use a Picard-like strategy, taking the viscosity evaluated with the velocity $\boldsymbol{u}^{n+\alpha, i}$ when 
$\boldsymbol{u}^{n+\alpha, i+1}$ is to be computed. Of course only a linear convergence rate can be expected if this is done, and the use of Newton's scheme for the convective terms seems unless. However, this depends on the relative importance of the non-linearities coming from the convective and the viscous terms. In some situations where the influence of the constitutive law is small and the flow is governed by convection, we have found useful to use $\lambda_{1}=1$ in Equation (13), even though the simple fixed-point scheme is used for the viscosity.

The convective term in the heat equation is another non-linearity of the problem. However, instead of linearizing this term and dealing with the fully coupled problem, with velocity, pressure and temperature as unknowns, we shall use an iterative coupling, as described, for example, in Reference [13]. The idea is to use the temperature known from the previous iteration in the momentum equation (10), and then use this equation and Equation (11) to compute the velocity and the pressure. With the velocity computed, we can proceed to solve the heat transport equation. Again, only a linear convergence rate can be expected for this iterative scheme. However, if the non-linear term of the momentum equation drives the iterative scheme, it can be useful to use $\lambda_{1}=1$ in Equation (13).

Having the previous considerations in mind, the fully linearized form of problem (10)-(12), coupling iteratively the heat equation to the momentum and incompressibility equations, is as follows: given a guess $\boldsymbol{u}^{n+\alpha, i}$ for $\boldsymbol{u}^{n+\alpha}$ and $\vartheta^{n+\alpha, i}$ for $\vartheta^{n+\alpha}$, find $\boldsymbol{u}^{n+\alpha, i+1} \in V^{n+\alpha}, p^{n+1, i+1} \in Q$ and $\vartheta^{n+\alpha, i+1} \in \Psi^{n+\alpha}$, such that
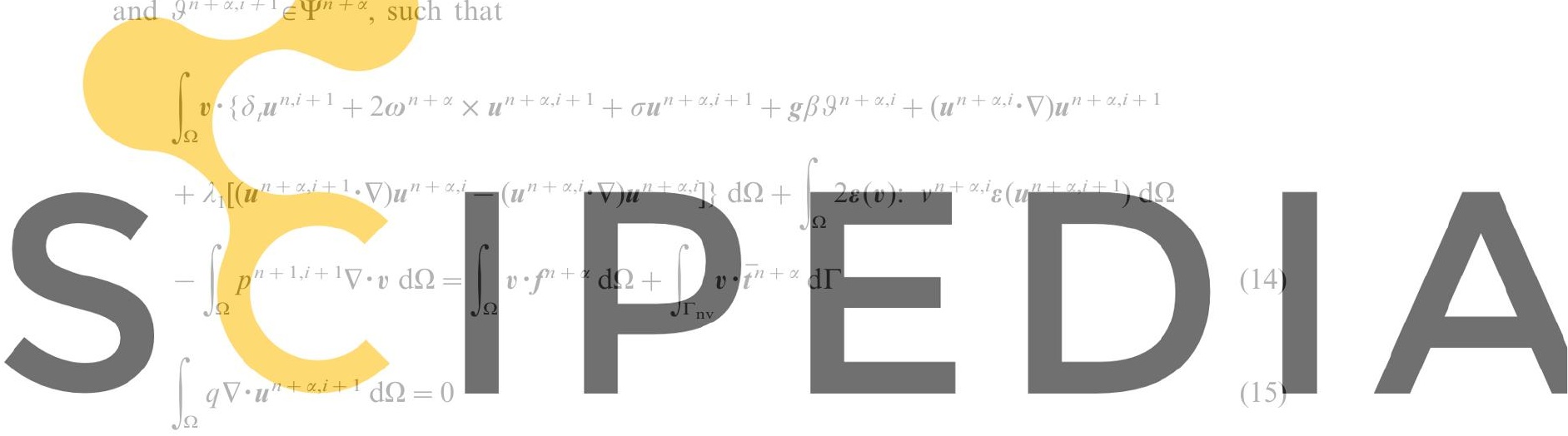

Register for free at https//www.scipedia.com to download the version without the watermar $\int_{\Omega} \psi\left[\delta_{t} \vartheta^{n, i+1}+\left(u^{n+\alpha, i+1} \cdot \nabla\right) \vartheta^{n+\alpha, i+1}\right] \mathrm{d} \Omega+\int_{\Omega} k \nabla \psi \cdot \nabla \vartheta^{n+\alpha, i+1} \mathrm{~d} \Omega$

$=\int_{\Omega} \psi S^{n+\alpha, i+1} \mathrm{~d} \Omega+\int_{\Gamma_{\mathrm{nt}}} \psi h^{n+\alpha} \mathrm{d} \Gamma$

for all test functions $v \in V_{0}, q \in Q$ and $\psi \in \Psi_{0}$. Here, $v^{n+\alpha, i}$ means that the viscosity is evaluated with $\boldsymbol{u}^{n+\alpha, i}$, whereas $S^{n+\alpha, i+1}$ is evaluated with $\boldsymbol{u}^{n+\alpha, i+1}$ ( $S$ can depend on $\boldsymbol{u}$ through Joule's effect, for instance). Likewise,

$$
\frac{\delta \boldsymbol{u}^{n, i+1}}{\delta t}=\frac{1}{\alpha \delta t}\left(\boldsymbol{u}^{n+\alpha, i+1}-\boldsymbol{u}^{n}\right)
$$


After problem (14)-(16) has been solved, convergence needs to be checked and, if not achieved, set $i \leftarrow i+1$ and solve this problem again.

\subsection{Finite element approximation}

We are now in a position to undertake the finite element approximation of the linear variational problem (14)-(16). In order to discretize it in space, let $\left\{\Omega^{e}\right\}$ be a finite element partition of the domain $\Omega$, with index $e$ ranging from 1 to the number of elements $n_{\mathrm{el}}$. We denote with a subscript $h$ the finite element approximation to the unknown functions, and by $\boldsymbol{v}_{h}, q_{h}$ and $\psi_{h}$ the velocity, pressure and temperature test functions associated to $\left\{\Omega^{e}\right\}$.

The finite element approximation to the functional spaces where the unknowns and the test functions belong are also characterized by a subscript $h$. A very important point is that we are interested in using equal interpolation for all the unknowns (velocity, pressure and temperature). Therefore, all the finite element spaces are assumed to be built-up using the standard continuous interpolation functions.

In order to overcome the numerical problems of the standard Galerkin method, a stabilized finite element formulation to solve (14)-(16) is applied. This formulation is presented in Reference [2] for the general case of systems of convection-diffusion-reaction equations, and applied to the incompressible Navier-Stokes equations in Reference [3], where its convergence properties for the linearized problem are analysed. The bottom line of the method is to test the continuous equations by the standard Galerkin test functions plus perturbations that depend on the operator representing the differential equation being solved. In our case, this operator corresponds to the linearized form of the time discrete Navier-Stokes equations and the heat equation. In this case, the method consists of finding $\boldsymbol{u}_{h}^{n+\alpha, i+1} \in V_{h}^{n+\alpha}, p_{h}^{n+1, i+1} \in Q_{h}$ and
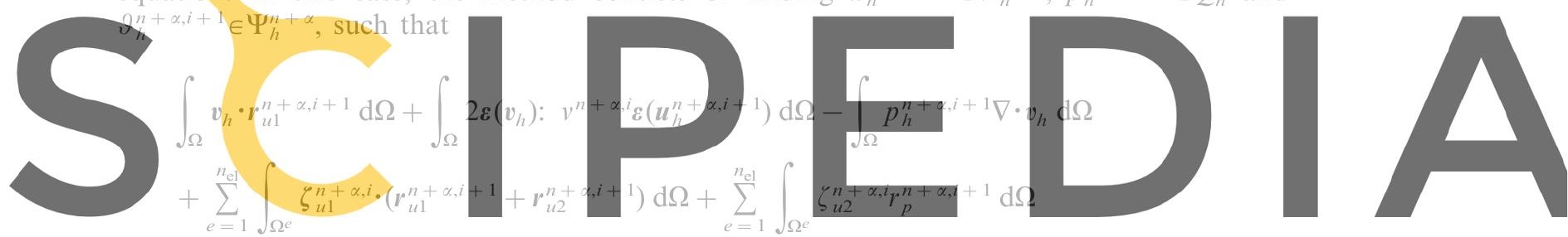

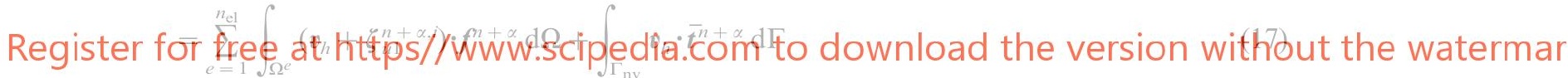

$$
\begin{aligned}
& \int_{\Omega} q_{h} r_{p}^{n+\alpha, i+1} \mathrm{~d} \Omega+\sum_{e=1}^{n_{\mathrm{el}}} \int_{\Omega^{e}} \zeta_{p}^{n+\alpha, i} \cdot\left(\boldsymbol{r}_{u 1}^{n+\alpha, i+1}+\boldsymbol{r}_{u 2}^{n+\alpha, i+1}\right) \mathrm{d} \Omega=\sum_{e=1}^{n_{\mathrm{el}}} \int_{\Omega^{e}} \zeta_{p}^{n+\alpha, i} \cdot \boldsymbol{f}^{n+\alpha} \mathrm{d} \Omega \\
& \int_{\Omega} \psi_{h} r_{\vartheta 1}^{n+\alpha, i+1} \mathrm{~d} \Omega+\int_{\Omega} \kappa \nabla \psi_{h} \cdot \nabla \vartheta_{h}^{n+\alpha, i+1} \mathrm{~d} \Omega+\sum_{e=1}^{n_{\mathrm{el}}} \int_{\Omega^{e}} \zeta_{p}^{n+\alpha, i}\left(r_{\vartheta 1}^{n+\alpha, i+1}+r_{\vartheta 2}^{n+\alpha, i+1}\right) \mathrm{d} \Omega \\
& =\sum_{e=1}^{n_{\mathrm{el}}} \int_{\Omega^{e}}\left(\psi_{h}+\zeta_{\vartheta}^{n+\alpha, i}\right) S^{n+\alpha, i+1} \mathrm{~d} \Omega+\int_{\Gamma_{\mathrm{nt}}} \psi_{h} h^{n+\alpha} \mathrm{d} \Gamma
\end{aligned}
$$

for all test functions $\boldsymbol{v}_{h} \in V_{0, h}, q_{h} \in Q_{h}$ and $\psi_{h} \in \Psi_{0, h}$, where 


$$
\begin{aligned}
\boldsymbol{r}_{u 1}^{n+\alpha, i+1}:= & \delta_{t} \boldsymbol{u}_{h}^{n, i+1}+2 \boldsymbol{\omega}^{n+\alpha} \times \boldsymbol{u}_{h}^{n+\alpha, i+1}+\sigma \boldsymbol{u}_{h}^{n+\alpha, i+1}+\boldsymbol{g} \beta \vartheta_{h}^{n+\alpha, i}+\left(\boldsymbol{u}_{h}^{n+\alpha, i} \cdot \nabla\right) \boldsymbol{u}_{h}^{n+\alpha, i+1} \\
& +\lambda_{1}\left[\left(\boldsymbol{u}_{h}^{n+\alpha, i+1} \cdot \nabla\right) \boldsymbol{u}_{h}^{n+\alpha, i}-\left(\boldsymbol{u}_{h}^{n+\alpha, i} \cdot \nabla\right) \boldsymbol{u}_{h}^{n+\alpha, i}\right] \\
\boldsymbol{r}_{u 2}^{n+\alpha, i+1}:= & -2 \nabla \cdot\left[v^{n+\alpha, i} \boldsymbol{g}\left(\boldsymbol{u}_{h}^{n+\alpha, i+1}\right)\right]+\nabla p_{h}^{n+1, i+1} \\
r_{p}^{n+\alpha, i+1}:= & \epsilon p_{h}^{n+1, i+1}-\lambda_{2} \epsilon p_{h}^{n+1, i}+\nabla \cdot \boldsymbol{u}_{h}^{n+\alpha, i+1} \\
r_{\vartheta 1}^{n+\alpha, i+1}:= & \delta_{t} \vartheta_{h}^{n, i+1}+\left(\boldsymbol{u}_{h}^{n+\alpha, i+1} \cdot \nabla\right) \vartheta_{h}^{n+\alpha, i+1} \\
r_{\vartheta 2}^{n+\alpha, i+1}:= & -\nabla\left(\kappa \cdot \nabla \vartheta_{h}^{n+\alpha, i+1}\right)
\end{aligned}
$$

the functions $\zeta_{u 1}, \zeta_{u 2}$ and $\zeta_{p}$ are computed within each element as

$$
\begin{aligned}
& \zeta_{u 1}=\tau_{u}\left\{\left(\boldsymbol{u}_{h} \cdot \nabla\right) \boldsymbol{v}_{h}+2 \boldsymbol{\omega} \times \boldsymbol{v}_{h}-\sigma \boldsymbol{v}_{h}+2 \nabla \cdot\left[v \boldsymbol{\varepsilon}\left(\boldsymbol{v}_{h}\right)\right]\right\} \\
& \zeta_{u 2}=\tau_{p} \nabla \cdot v_{h} \\
& \zeta_{p}=\tau_{u} \nabla q_{h} \\
& \zeta_{9}=\tau_{g}\left[\left(\boldsymbol{u}_{h} \cdot \nabla\right) \psi_{h}+\nabla \cdot\left(\kappa \nabla \psi_{h}\right)\right]
\end{aligned}
$$

and the parameters $\tau_{u}, \tau_{p}$ and $\tau_{\vartheta}$ are also computed elementwise as $[3,14]$
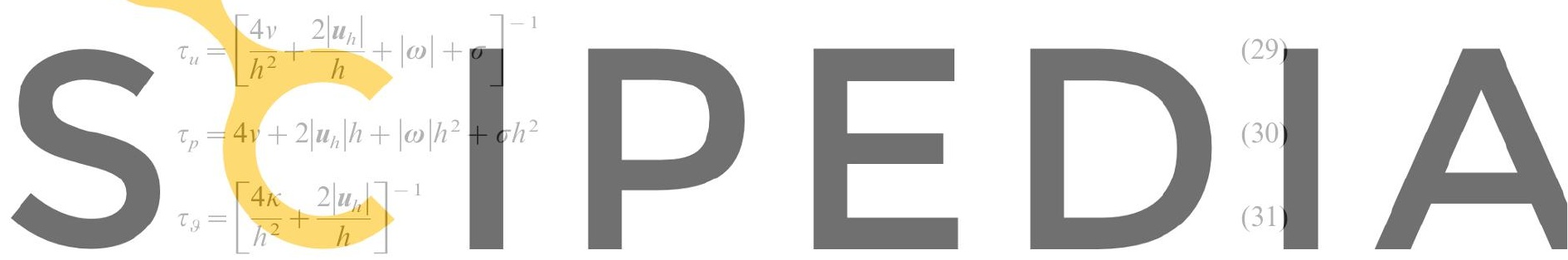

where $h$ is the element size for linear elements and half of it for quadratics.

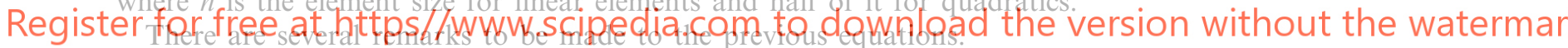

Remark 1

It is observed that Equations (21) and (24) (the terms of the original differential equations integrated by parts in the weak form of the problem) involve second derivatives of the unknowns. This is why the integrals involving these terms have to be evaluated element by element.

\section{Remark 2}

The term (21) also involves derivatives of the viscosity, in the case in which it is variable. These are very difficult to incorporate in the formulation, although in the next section it is discussed how to deal with the viscosity variation in the nodal-based implementation presented there. 


\section{Remark 3}

In Equation (22) we have introduced a parameter $\epsilon$ that corresponds to a penalty parameter for the incompressibility constraint. When $\lambda_{2}=0$, the penalty strategy can be considered the 'classical' one. On the other hand, when $\lambda_{2}=1$ it is seen from Equation (22) that the effect of the penalization disappears when convergence is achieved. This iterative penalty method is discussed and analysed in Reference [15]. The benefit of taking $\lambda_{2}=1$ is that larger values of $\epsilon$ may be used with a good approximation of the incompressibility constraint. The use of penalty methods is very useful when pressures are discontinuous, since they can be eliminated at the element level. When continuous pressures are used, they may help to improve the convergence of the iterative methods if they are used to solve the algebraic system of equations.

\section{Remark 4}

In expressions (25)-(31) the velocity $\boldsymbol{u}_{h}$, the speed of rotation $\omega$ and the viscosity $v$ are evaluated at the step and iteration indicated in (17)-(24).

\section{Pemark 5}

It is observed from (25)-(28) that these terms are precisely the adjoints of the (linearized) operators of the differential equations to be solved applied to the test functions (observe the signs of the yiscous and permeability terms in Equation (25) and of the diffusive term in Equation (28)). This method corresponds to the algebraic version of the sub-grid scale approach [1,2] and circumvents all the stability problems of the Galerkin method. In particular, in this case it is possible to use equal velocity-pressure interpolations, i.e. we are not tight to the satisfaction of the inf-sup stability condition.
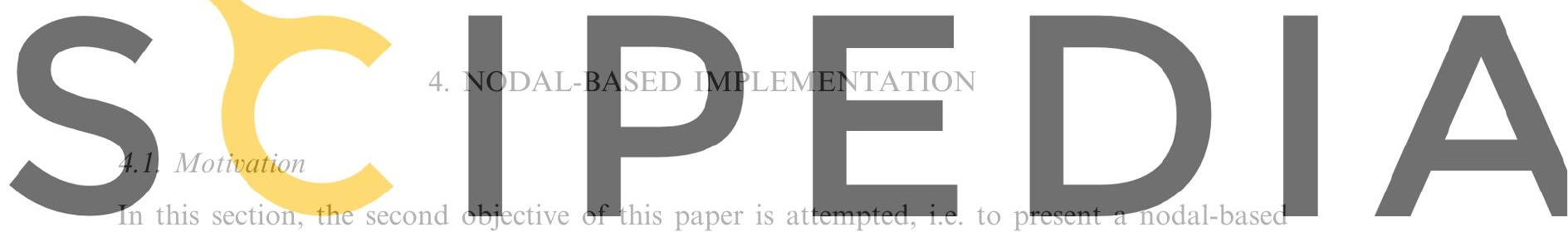

finite element implementation of the stabilized finite element formulation presented in the

Registerefopk freediat https//www.scipedia.com to download the version without the watermar

Let $n_{\mathrm{pts}}$ be the total number of nodes of the finite element mesh and let $N^{a}$ be the shape

function (i.e. the standard finite element interpolation function) associated with node $a$;

$a=1, \ldots, n_{\mathrm{pts}}$. From now on, superscripts $a$ and $b$ will refer to the nodes of the mesh.

To obtain the algebraic version of problem (17)-(19), the standard procedure is to interpolate the unknowns as

$$
\begin{aligned}
& u_{h, i}=\sum_{a=1}^{n_{\mathrm{pts}}} N^{a} U_{i}^{a}, \quad i=1, \ldots, n_{\mathrm{sd}} \\
& p_{h}=\sum_{a=1}^{n_{\mathrm{pts}}} N^{a} P_{i}^{a}
\end{aligned}
$$




$$
\vartheta_{h}=\sum_{a=1}^{n_{\mathrm{pts}}} N^{a} \Theta^{a}
$$

where upper characters $U, P$ and $\Theta$ are used to denote the nodal values of the corresponding lower case variables (at the time step and iteration of interest). The test functions are then taken as $v_{h, i}=N^{b} \delta_{i k}$ for $k=1, \ldots, n_{\text {sd }}$ ( $\delta_{i k}$ being the Kronecker delta), $q_{h}=N^{b}$ and $\psi_{h}=N^{b}$, $b=1, \ldots, n_{\mathrm{pts}}$. After the boundary conditions of Dirichlet type are prescribed, this leads to an algebraic system of equations the solution of which yields the nodal unknowns. The matrix of this algebraic system changes from time step to time step and from iteration to iteration due to three reasons: the convective term, the viscous term and the stabilization parameters given in Equations (29)-(31). All these terms depend on the velocity, and hence on the iteration and time step. The former dependence could be avoided by treating explicitly in time these terms, but this would be at the expense of loosing stability of the time integration.

The time consuming task in the calculation of the matrix of the algebraic system (traditionally referred to as 'stiffness matrix') is the numerical integration involved. However, it is possible to introduce some approximations that allow the expression of all the integral in terms of

$$
\begin{aligned}
& \int_{\Omega} N^{a} N^{b} \mathrm{~d} \Omega \\
& \int_{\Omega} N^{a} \partial_{i} N^{b} \mathrm{~d} \Omega, \quad \int_{\Omega} \partial_{i} N^{a} N^{b} \mathrm{~d} \Omega, \quad i=1, \ldots, n_{\mathrm{sd}} \\
& \int_{\Omega} \partial_{i} N^{a} \partial_{j} N^{b} \mathrm{~d} \Omega, \quad i, j=1, \ldots, n_{\mathrm{sd}}
\end{aligned}
$$

for $a, b=1, \ldots, n_{\mathrm{pts}}$, and, if the second derivatives of the shape functions within an element are not zero (or negligible), also in terms of

$$
\begin{array}{ll}
\sum_{e=1}^{n_{\mathrm{el}}} \int_{\Omega^{e}} N^{a} \Delta N^{b} \mathrm{~d} \Omega, & \sum_{e=1}^{n_{\mathrm{el}}} \int_{\Omega^{e}} \Delta N^{a} N^{b} \mathrm{~d} \Omega \\
\sum_{e=1}^{n_{\mathrm{el}}} \int_{\Omega^{e}} \Delta N^{a} \partial_{i} N^{b} \mathrm{~d} \Omega, & \sum_{e=1}^{n_{\mathrm{el}}} \int_{\Omega^{e}} \partial_{i} N^{a} \Delta N^{b} \mathrm{~d} \Omega, \quad i=1, \ldots, n_{\mathrm{sd}} \\
\sum_{e=1}^{n_{\mathrm{el}}} \int_{\Omega^{e}} \Delta N^{a} \Delta N^{b} \mathrm{~d} \Omega &
\end{array}
$$

for $a, b=1, \ldots, n_{\mathrm{pts}}$. In the previous expressions, $\partial_{i}$ denotes the partial derivative with respect to the $i$ th Cartesian co-ordinate, and $\Delta$ is the Laplacian operator. For fixed domains $\Omega$, all the integrals in Equations (32) and (33) can be computed at the beginning of the run and stored.

At this point there are two questions to be treated. This first is which are the approximations needed to be really able to use only Equations (32) and (33) to build up the matrix of the algebraic system. This is the subject of Sections 4.2 and 4.3 . 
The second question is how to store the integrals in Equations (32) and (33). The efficiency of the overall implementation depends on how efficient the storage scheme is. Let us only mention that the technique adopted in this work is to use a compressed sparse row (CSR) format to store the $n_{\mathrm{pts}} \times n_{\mathrm{pts}}$ matrix of the graph associated to the finite element mesh.

\subsection{Approximation of the viscous and convective terms}

As it has been mentioned before, the convective and the viscous terms (when the viscosity depends on the velocity) need to be recomputed at each iteration of each time step. However, it is possible to approximate these two terms so that they can be computed with the integrals appearing in (32).

4.2.1. Convective term. Let us begin with the convective term in Equation (17) and assuming for simplicity a Picard linearization, i.e. $\lambda_{1}=0$. The terms appearing when $\lambda_{1}=1$, as well as the convective term in the discrete heat equation (19), can be dealt with in a similar way.

Let $\boldsymbol{a}_{h} \equiv \boldsymbol{u}_{h}^{n+\alpha, i}$ and $\boldsymbol{u}_{h} \equiv \boldsymbol{u}_{h}^{n+\alpha, i+1}$. When the velocity test function is taken such that $v_{h, i}=N^{b} \delta_{i k}$, with $k$ fixed $\left(k=1, \ldots, n_{\text {sd }}\right)$, the convective term is

$$
\int_{\Omega} \boldsymbol{v}_{h} \cdot\left[\left(\boldsymbol{a}_{h} \cdot \nabla\right) \boldsymbol{u}_{h}\right] \mathrm{d} \Omega=\sum_{j=1}^{n_{\mathrm{sd}}}\left(\int_{\Omega} N^{b} a_{h, j} \partial_{j} N^{a} \mathrm{~d} \Omega\right) U_{k}^{a}
$$

The need for an additional approximation arises because of the function $a_{h, j}$ appearing within the integrals. Calling $A_{j}^{a}$ the nodal values of this function, the approximation that is proposed here is

$$
\sum_{j=1}^{n_{\mathrm{sd}}}\left(\int_{\Omega} N^{b} a_{h, j} \partial_{j} N^{a} \mathrm{~d} \Omega\right) \approx \sum_{j=1}^{n_{\mathrm{sd}}} A_{j}^{c}\left(\int_{\Omega} N^{b} \partial_{j} N^{a} \mathrm{~d} \Omega\right)
$$

where $c=b$ or $c=a$. In any case, the convective term will be expressed in terms of the integrals of Equations (32), as desired. The reasons for one choice or the other are discussed next.

Choice $c=b$ in (35). Suppose that there exists a nodal integration rule of order $n_{\text {int }}$. The integration points are then the nodes of the mesh, of co-ordinates $\boldsymbol{x}^{g}, g=1, \ldots, n_{\mathrm{pts}}$. The associated weights for the $e$ th element are denoted $W_{e}^{g}$ and the number of nodes per element by $n_{\text {nod }}$ (the same superscript $g$ is used for the numbering of the element nodes). Interpolating the velocity components in the left-hand side of (35) and using the fact that $N^{a}\left(\boldsymbol{x}^{b}\right)=\delta^{a b}$, we have

$$
\begin{aligned}
\sum_{j=1}^{n_{\mathrm{sd}}}\left(\int_{\Omega} N^{b} a_{h, j} \partial_{j} N^{a} \mathrm{~d} \Omega\right) & =\sum_{j=1}^{n_{\mathrm{sd}}} \sum_{c=1}^{n_{\mathrm{pts}}} A_{j}^{c}\left(\int_{\Omega} N^{b} N^{c} \partial_{j} N^{a} \mathrm{~d} \Omega\right) \\
& =\sum_{j=1}^{n_{\mathrm{sd}}} \sum_{c=1}^{n_{\mathrm{pts}}} A_{j}^{c}\left(\left.\sum_{e=1}^{n_{\mathrm{el}}} \sum_{g=1}^{n_{\mathrm{nod}}} W_{e}^{g} \delta^{b g} \delta^{c g} \partial_{j} N^{a}\left(\boldsymbol{x}^{g}\right)\right|_{\Omega^{e}}\right)+O\left(h^{n_{\mathrm{int}}}\right)
\end{aligned}
$$




$$
\begin{aligned}
& =\sum_{j=1}^{n_{\mathrm{sd}}} A_{j}^{b}\left(\left.\sum_{e=1}^{n_{\mathrm{el}}} W_{e}^{b} \partial_{j} N^{a}\left(\boldsymbol{x}^{b}\right)\right|_{\Omega^{e}}\right)+O\left(h^{n_{\mathrm{int}}}\right) \\
& =\sum_{j=1}^{n_{\mathrm{sd}}} A_{j}^{b}\left(\int_{\Omega} N^{b} \partial_{j} N^{a} \mathrm{~d} \Omega\right)+O\left(h^{n_{\mathrm{int}}}\right)
\end{aligned}
$$

which justifies the use of (35) with $c=b$. It is seen that the error is $O\left(h^{n_{\mathrm{int}}}\right)$. It is known that the nodal numerical integration is of order $n_{\mathrm{int}}=p+1$, where $p$ is the order of the standard Lagrange interpolation. Therefore, the error of approximation (35) is the same as the order of the finite element interpolation when $c=b$. However, the argument used to arrive to this conclusion is not valid for the stabilization term, whereas the following can also be applied in this case, as will be shown later.

Choice $c=a$ in (35). Since $\boldsymbol{a}_{h}$ is approximately divergence free, we can approximate

$$
\int_{\Omega} \boldsymbol{v}_{h} \cdot\left[\left(\boldsymbol{a}_{h} \cdot \nabla\right) \boldsymbol{u}_{h}\right] \mathrm{d} \Omega \approx \int_{\Omega} \boldsymbol{v}_{h} \cdot\left[\nabla \cdot\left(\boldsymbol{a}_{h} \otimes \boldsymbol{u}_{h}\right)\right] \mathrm{d} \Omega
$$

In fact, it is not necessary to consider this as an approximation, since the convective term of the original continuous equations could have been written directly as $\nabla \cdot(\boldsymbol{u} \otimes \boldsymbol{u})$ rather than $(\boldsymbol{u} \cdot \nabla) \boldsymbol{u}$. What is definitely an approximations to interpolate the product $\boldsymbol{a}_{h} \otimes \boldsymbol{u}_{h}$ instead of each of the components separately. Doing this when $v_{h, i}=N^{b} \delta_{i k}$ yields

$$
\sum_{i, j=1}^{n_{\mathrm{sd}}} \int_{\Omega} v_{h, i} \partial_{j}\left(a_{h, j} u_{h, i}\right) \mathrm{d} \Omega \approx \sum_{j=1}^{n_{\mathrm{sd}}} \int_{\Omega} N^{b} \partial_{j}\left(\sum_{a=1}^{n_{\mathrm{pts}}} N^{a} A_{j}^{a} U_{k}^{a}\right) \mathrm{d} \Omega=\sum_{j=1}^{n_{\mathrm{sd}}} \sum_{a=1}^{n_{\mathrm{pts}}} A_{j}^{a}\left(\int_{\Omega} N^{b} \partial_{j} N^{a} \mathrm{~d} \Omega\right) U_{k}^{a}
$$

which justifies (35) for $c=a$. The performance of this approximation, as well of the following, will be checked through numerical experiments. However, it can be anticipated that a certain loss of accuracy can occur, since piecewise polynomial solutions of order $p$ will not anymore be a solution of the discrete problem when elements of order $p$ are used, since for such solutions $\boldsymbol{u} \otimes \boldsymbol{u}$ is a polynomial of order $2 p$.

4.2.2. Viscous term. Let us consider now the approximation of the viscous term in Equation (17). When $v_{h, i}=N^{b} \delta_{i k}$, with $k$ fixed, this term is

$$
2 \int_{\Omega} \boldsymbol{\varepsilon}\left(\boldsymbol{v}_{h}\right): v \boldsymbol{\varepsilon}\left(\boldsymbol{u}_{h}\right) \mathrm{d} \Omega=\sum_{i, j=1}^{n_{\mathrm{sd}}} \int_{\Omega} v \partial_{i} v_{h, j}\left(\partial_{i} u_{h, j}+\partial_{j} u_{h, i}\right) \mathrm{d} \Omega=\sum_{i=1}^{n_{\mathrm{sd}}} \int_{\Omega_{b}} v \partial_{i} N^{b}\left(\partial_{i} u_{h, k}+\partial_{k} u_{h, i}\right) \mathrm{d} \Omega
$$

where $\Omega_{b}$ is the interior of the support of $N^{b}$, i.e. the union of the domains of the elements to which node $b$ belongs. To see how these integrals can be approximated, let $f$ and $g$ be two given functions, both bounded and the former with bounded derivatives. Expanding $f$ in Taylor series, the integral of their product in a domain $\Omega_{0}$ is 


$$
\begin{aligned}
\int_{\Omega_{0}} f g \mathrm{~d} \Omega= & f(\overline{\boldsymbol{x}}) \int_{\Omega_{0}} g \mathrm{~d} \Omega+\left.\sum_{i=1}^{n_{\mathrm{sd}}} \partial_{i} f\right|_{\overline{\boldsymbol{x}}} \int_{\Omega_{0}}\left(x_{i}-\bar{x}_{i}\right) g \mathrm{~d} \Omega \\
& +\left.\frac{1}{2} \sum_{i, j=1}^{n_{\mathrm{sd}}} \partial_{i} \partial_{j} f\right|_{\overline{\boldsymbol{x}}} \int_{\Omega_{0}}\left(x_{i}-\bar{x}_{i}\right)\left(x_{j}-\bar{x}_{j}\right) g \mathrm{~d} \Omega+\cdots
\end{aligned}
$$

If $d$ is the diameter of $\Omega_{0}$ and meas $\left(\Omega_{0}\right)$ its measure, we see that

$$
\int_{\Omega_{0}} f g \mathrm{~d} \Omega=f(\overline{\boldsymbol{x}}) \int_{\Omega_{0}} g \mathrm{~d} \Omega+O\left(d^{m} \operatorname{meas}\left(\Omega_{0}\right)\right)
$$

where $m=2$ if $\overline{\boldsymbol{x}}$ is the centre of mass of $\Omega_{0}$ with 'density' $g$ and $m=1$ otherwise. The idea is now to apply this to the integrals in (39), with $f \equiv v$ and $g \equiv \partial_{i} N^{b}\left(\partial_{i} u_{h, k}+\partial_{k} u_{h, i}\right)$. Obviously, it would be desirable to know the centre of mass of $\Omega_{b}$, with density function $g$, which is unknown. What is proposed here is to take $v$ in Equation (39) as constant and equal to its value $v^{b}$ at node $b$. Doing this and interpolating the velocity yields

$$
\begin{aligned}
\sum_{i=1}^{n_{\mathrm{sd}}} \int_{\Omega_{b}} v \partial_{i} N^{b}\left(\partial_{i} u_{h, k}+\partial_{k} u_{h, i}\right) \mathrm{d} \Omega \approx & \sum_{i=1}^{n_{\mathrm{sd}}} v^{b} \int_{\Omega_{b}} \partial_{i} N^{b}\left(\partial_{i} u_{h, k}+\partial_{k} u_{h, i}\right) \mathrm{d} \Omega \\
= & \sum_{i=1}^{n_{\mathrm{sd}}} \sum_{a=1}^{n_{\mathrm{pts}}}\left[v^{b}\left(\int_{\Omega} \partial_{i} N^{b} \partial_{i} N^{a} \mathrm{~d} \Omega\right) U_{k}^{a}\right. \\
& \left.+v^{b}\left(\int_{\Omega} \partial_{i} N^{b} \partial_{k} N^{a} \mathrm{~d} \Omega\right) U_{i}^{a}\right]
\end{aligned}
$$

Again, this expression involves only the integrals in (32).

The way to evaluate $v^{b}$ is not absolutely clear. For constitutive laws such as (5), the viscosity depends on the velocity gradients, which are discontinuous for standard $C^{0}$ finite element interpolations. In order to obtain nodal values of these velocity gradients, we use a standard least-square smoothing from the elementwise values. If $G_{i j}^{a}, a=1, \ldots, n_{\mathrm{pts}}$, are the nodal values of $\partial_{i} u_{h, j}, i, j=1, \ldots, n_{\mathrm{sd}}$, these are the solution of the linear system

$$
\sum_{a=1}^{n_{\mathrm{pts}}}\left(\int_{\Omega} N^{b} N^{a} \mathrm{~d} \Omega,\right) G_{i j}^{a}=\sum_{a=1}^{n_{\mathrm{pts}}}\left(\int_{\Omega} N^{b} \partial_{i} N^{a} \mathrm{~d} \Omega,\right) U_{j}^{a}, \quad b=1, \ldots, n_{\mathrm{pts}}
$$

which only involves the integrals in (32). Moreover, to avoid the need for solving (42), we use a nodal numerical integration rule to compute the integral of the left-hand side, yielding a diagonal 'mass' matrix. Also, since for quadratic elements there are weights which are zero, they are replaced by those corresponding to the splitting of the quadratic elements into a number of linear elements. 


\section{Remark 6}

Since

$$
\int_{\Omega} \partial_{i} N^{b} \partial_{i} N^{a} \mathrm{~d} \Omega=\int_{\Omega_{b} \cap \Omega_{a}} \partial_{i} N^{b} \partial_{i} N^{a} \mathrm{~d} \Omega
$$

it is tempting to evaluate the viscosity at a point in $\Omega_{b} \cap \Omega_{a}$, not necessarily node $b$. However, if $v$ depends also on node $a$, the second equality in (41) does not hold and the viscous term would be wrongly approximated, since for 'exact' nodal values $U_{k}^{a}$ the discrete variational equation (17) would not be satisfied. This can be understood as a lack of 'consistency' of the numerical formulation. In practice, what we have observed is a completely oscillating behaviour of the iterative scheme, leading to converged solutions only for mild nonlinearities.

\subsection{Nodal stabilization parameters: consistency and conservation}

The last point that needs to be analysed is the way in which the stabilization terms appearing in (17)-(19) can be approximated to achieve the goal of using only the integrals in (32) and (33) in the implementation. For the purpose of this section, it suffices to consider the stationary problem with $\omega=\mathbf{0}$ and $\sigma=0$. Also, $\lambda_{1}=\lambda_{2}=0$ are taken. Likewise, attention shall be focused on the Navier-Stokes equations, although the same ideas can be applied to the heat equation.

As before, let $\boldsymbol{a}_{h}$ be the velocity of the previous iteration and $\boldsymbol{u}_{h}$ the velocity field that needs to be computed. The first approximation to be considered refers to the viscous term of the element residual in (17). Using the fact that the exact velocity is divergence free, the approximation

$$
\left.\left.2 \nabla \cdot\left[v \boldsymbol{\varepsilon}\left(\boldsymbol{u}_{h}\right)\right]\right|_{\Omega^{e}} \approx v \Delta \boldsymbol{u}_{h}\right|_{\Omega^{e}}+\left.2 \nabla v \cdot \boldsymbol{\varepsilon}\left(\boldsymbol{u}_{h}\right)\right|_{\Omega^{e}}
$$

avoids the need for computing and storing all the second derivatives of the shape functions, and only their Laplacian needs to be dealt with. Observe that this does not affect the natural boundary conditions associated with the discrete weak problem, since it is used only for the elementwise evaluation of the viscous term. To simplify the calculations, the second term in (44) is evaluated at the previous iteration, computing nodal values for it by using a least-squares smoothing for the viscosity gradients and the velocity gradients as in (42). These nodal values are then added to those of the external force $f$, which is considered to account for them in the following.

Using approximation (44) and considering the simplified situation described above, the discrete problem to be solved is

$$
\begin{aligned}
& \int_{\Omega} \boldsymbol{v}_{h} \cdot\left[\left(\boldsymbol{a}_{h} \cdot \nabla\right) \boldsymbol{u}_{h}\right] \mathrm{d} \Omega+2 \int_{\Omega} \boldsymbol{\varepsilon}\left(\boldsymbol{v}_{h}\right): v \boldsymbol{\varepsilon}\left(\boldsymbol{u}_{h}\right) \mathrm{d} \Omega-\int_{\Omega} p_{h} \nabla \cdot \boldsymbol{v}_{h} \mathrm{~d} \Omega+S_{\mathrm{mom}, 1}\left(\boldsymbol{v}_{h} ; \boldsymbol{u}_{h}, p_{h}\right) \\
& +S_{\mathrm{mom}, 2}\left(\boldsymbol{v}_{h} ; \boldsymbol{u}_{h}\right)=R_{\text {mom }}\left(\boldsymbol{v}_{h}\right)
\end{aligned}
$$




$$
\epsilon \int_{\Omega} p_{h} q_{h} \mathrm{~d} \Omega+\int_{\Omega} q_{h} \nabla \cdot \boldsymbol{u}_{h}+S_{\mathrm{cont}}\left(q_{h} ; \boldsymbol{u}_{h}, p_{h}\right)=R_{\mathrm{cont}}\left(q_{h}\right)
$$

where the right-hand side terms $R_{\text {mom }}\left(v_{h}\right)$ and $R_{\text {cont }}\left(q_{h}\right)$ come from the force vector (4) and the surface traction in (7)

$$
\begin{aligned}
& R_{\mathrm{mom}}\left(\boldsymbol{v}_{h}\right):=\int_{\Omega} \boldsymbol{v}_{h} \cdot \boldsymbol{f} \mathrm{d} \Omega+\int_{\Gamma_{n v}} \boldsymbol{v}_{h} \cdot \overline{\boldsymbol{t}} \mathrm{d} \Gamma+\sum_{e=1}^{n_{\mathrm{el}}} \int_{\Omega^{\mathrm{e}}} \tau_{u}\left[\left(\boldsymbol{a}_{h} \cdot \nabla\right) \boldsymbol{v}_{h}+v \Delta \boldsymbol{v}_{h}\right] \cdot \boldsymbol{f} \mathrm{d} \Omega \\
& R_{\mathrm{cont}}\left(q_{h}\right):=\sum_{e=1}^{n_{\mathrm{el}}} \int_{\Omega^{e}} \tau_{u} \nabla q_{h} \cdot \boldsymbol{f} \mathrm{d} \Omega
\end{aligned}
$$

and the stabilization terms are given by

$$
\begin{aligned}
& S_{\mathrm{mom}, 1}\left(\boldsymbol{v}_{h} ; \boldsymbol{u}_{h}, p_{h}\right)=\sum_{e=1}^{n_{\mathrm{el}}} \int_{\Omega^{e}} \tau_{u}\left[\left(\boldsymbol{a}_{h} \cdot \nabla\right) \boldsymbol{v}_{h}+v \Delta \boldsymbol{v}_{h}\right] \cdot\left[\left(\boldsymbol{a}_{h} \cdot \nabla\right) \boldsymbol{u}_{h}-v \Delta \boldsymbol{u}_{h}+\nabla p_{h}\right] \mathrm{d} \Omega \\
& S_{\mathrm{mom}, 2}\left(\boldsymbol{v}_{h} ; \boldsymbol{u}_{h}\right)=\sum_{e=1}^{n_{\mathrm{el}}} \int_{\Omega^{e}} \tau_{p}\left(\nabla \cdot \boldsymbol{v}_{h}\right)\left(\nabla \cdot \boldsymbol{u}_{h}\right) \mathrm{d} \Omega \\
& S_{\mathrm{cont}}\left(q_{h} ; \boldsymbol{u}_{h}, p_{h}\right)=\sum_{e=1}^{n_{\mathrm{el}}} \int_{\Omega^{e}} \tau_{u} \nabla q_{h} \cdot\left[\left(\boldsymbol{a}_{h} \cdot \nabla\right) \boldsymbol{u}_{h}-v \Delta \boldsymbol{u}_{h}+\nabla p_{h}\right] \mathrm{d} \Omega
\end{aligned}
$$

where the stabilization parameters $\tau_{u}$ and $\tau_{p}$ are given by (29) and (30) respectively.

Suppose for a moment that $\Gamma_{\mathrm{nv}}=\partial \Omega$, i.e. all the boundary conditions are of Neumann type (case in which the solution would not be unique) and that we can take the test function $\boldsymbol{v}_{h}$ constant. Assuming that $\boldsymbol{a}_{h}$ is divergence free (or using expression (37) for the convective term) equations (45)-(46) imply in this case

$$
\begin{aligned}
& \int_{\partial \boldsymbol{\Omega}}\left(\boldsymbol{n} \cdot \boldsymbol{a}_{h}\right) \boldsymbol{u}_{h} \mathrm{~d} \Omega=\int_{\Omega} \boldsymbol{f} \mathrm{d} \Omega+\int_{\partial \boldsymbol{\Omega}} \overline{\boldsymbol{t}} \mathrm{d} \Gamma \\
& \epsilon \int_{\Omega} p_{h} \mathrm{~d} \Omega+\int_{\partial \boldsymbol{\Omega}} \boldsymbol{n} \cdot \boldsymbol{u}_{h} \mathrm{~d} \Gamma=0
\end{aligned}
$$

which can be understood as global conservation statements for the momentum and the mass of the fluid contained in the domain $\Omega$. It is in this sense that the finite element method can be considered as 'conservative'.

However, Equations (45) and (46) are not enforced for constant test functions $\boldsymbol{v}_{h}$ and $q_{h}$, but only for test functions of the form $\boldsymbol{v}_{h}=N^{b} \boldsymbol{e}_{k}$ and $q_{h}=N^{b}, b=1, \ldots, n_{\mathrm{sd}}$, where $\boldsymbol{e}_{k}$ is the unit vector along the $x_{k}$ co-ordinate. Since the addition of all the shape functions $N^{b}$ is 1 , Equations (50) and (51) can also be obtained by adding up, for $b=1$ to $b=n_{\mathrm{pts}}$, Equation (45) enforced for $\boldsymbol{v}_{h}=N^{b} \boldsymbol{e}_{k}$ and also (46) enforced for $q_{h}=N^{b}$, provided that 


$$
\begin{aligned}
& \sum_{b=1}^{n_{\text {pts }}} S_{\text {mom }, 1}\left(N^{b} \boldsymbol{e}_{k} ; \boldsymbol{u}_{h}, p_{h}\right)=0 \\
& \sum_{b=1}^{n_{\text {pts }}} S_{\text {mom }, 2}\left(N^{b} \boldsymbol{e}_{k} ; \boldsymbol{u}_{h}\right)=0 \\
& \sum_{b=1}^{n_{\text {pts }}} S_{\text {cont }}\left(N^{b} ; \boldsymbol{u}_{h}, p_{h}\right)=0
\end{aligned}
$$

These equations can be considered as the sufficient conditions for the stabilized finite element method to be conservative (see Reference [14] for further discussion).

In a standard implementation of the formulation, the stabilization parameters are computed depending only on the element and not on the test functions being used in (47)-(49). However, the idea of the nodal-based implementation presented here is to use nodal values for all the parameters of the formulation and in particular for $\tau_{u}$ and $\tau_{p}$. It can be readily observed from (47)-(49) for $\boldsymbol{v}_{h}=N^{b} \boldsymbol{e}_{k}$ that conditions (52)-(54) will not hold if $\tau_{u}$ and $\tau_{p}$ depend on $b$. If this happens it is impossible to assess that the numerical formulation is conservative.

Of special interest is what happens to the continuity equation (46) when a penalty parameter is used. Taking $q_{h}=N^{b}$ and adding up for all $b$ yields

$$
\sum_{b=1}^{n_{\text {pts }}} \sum_{e=1}^{n_{\text {el }}} \int_{\Omega^{e}} \tau_{u} \nabla_{u} N^{b} \cdot\left[\left(\boldsymbol{a}_{h} \cdot \nabla\right) \boldsymbol{u}_{h}-v \Delta \boldsymbol{u}_{h}+\nabla p_{h}\right] \mathrm{d} \Omega+\epsilon \int_{\Omega} p_{h} \mathrm{~d} \Omega+\int_{\partial \Omega} \boldsymbol{n} \cdot \boldsymbol{u}_{h} \mathrm{~d} \Gamma=0
$$

If $\tau_{\mathrm{u}}$ is independent of $b$ the first term is zero and thus the mean pressure value is zero when so is the mass flux in the domain $\Omega$. This is an interesting property of penalty methods that does not hold when $\tau_{u}$ changes according to different values of $b$, since the first term is not necessarily zero in this case. If the mass flux is zero (for instance, because of a Dirichlet prescription for the velocity), the outcome is that the smaller the value of $\epsilon$ is, the larger the mean pressure value. This behaviour has been observed in numerical experiments.

Despite this lack of 'conservation', which has to be acknowledged, the stabilization parameters $\tau_{u}$ and $\tau_{p}$ will be evaluated at node $b$ when $\boldsymbol{v}_{h}=N^{b} \boldsymbol{e}_{k}$ and when $q_{h}=N^{b}$. The reason for this is related to the consistency of the scheme, which is discussed next.

Let us consider the stabilization term (47). Taking $\boldsymbol{v}_{h}=N^{b} \boldsymbol{e}_{k}$ and interpolating the velocity and the pressure it is found that

$$
\begin{aligned}
S_{\mathrm{mom}, 1}\left(N^{b} \boldsymbol{e}_{k} ; \boldsymbol{u}_{h}, p_{h}\right)= & \sum_{e=1}^{n_{\mathrm{el}}} \int_{\Omega^{e}} \tau_{u}\left[\sum_{i=1}^{n_{\mathrm{sd}}}\left(a_{h, i} \partial_{i} N^{b}\right)+v \Delta N^{b}\right] \\
& \cdot\left[\sum_{a=1}^{n_{\mathrm{pts}}} \sum_{j=1}^{n_{\mathrm{sd}}}\left(a_{h, j} \partial_{j} N^{a}\right) U_{k}^{a}-v \sum_{a=1}^{n_{\mathrm{pts}}} \Delta N^{a} U_{k}^{a}+\sum_{a=1}^{n_{\mathrm{pts}}} \partial_{k} N^{a} P^{a}\right] \mathrm{d} \Omega \\
= & \sum_{a=1}^{n_{\mathrm{pts}}}\left[\sum_{i, j=1}^{n_{\mathrm{sd}}} \int_{\Omega} \tau_{u}\left(a_{h, i} \partial_{i} N^{b}\right)\left(a_{h, j} \partial_{j} N^{a}\right) \mathrm{d} \Omega\right] U_{k}^{a} \\
& -\sum_{a=1}^{n_{\mathrm{pts}}}\left[\sum_{i=1}^{n_{\mathrm{sd}}} \sum_{e=1}^{n_{\mathrm{el}}} \int_{\Omega^{e}} \tau_{u} v\left(a_{h, i} \partial_{i} N^{b}\right) \Delta N^{a} \mathrm{~d} \Omega\right] U_{k}^{a}
\end{aligned}
$$




$$
\begin{aligned}
& +\sum_{a=1}^{n_{\mathrm{pts}}}\left[\sum_{i=1}^{n_{\mathrm{sd}}} \int_{\Omega} \tau_{u}\left(a_{h, i} \partial_{i} N^{b}\right) \partial_{k} N^{a} \mathrm{~d} \Omega\right] P^{a} \\
& +\sum_{a=1}^{n_{\mathrm{pts}}}\left[\sum_{j=1}^{n_{\mathrm{sd}}} \sum_{e=1}^{n_{\mathrm{el}}} \int_{\Omega^{e}} \tau_{u} v \Delta N^{b}\left(a_{h, j} \partial_{j} N^{a}\right) \mathrm{d} \Omega\right] U_{k}^{a} \\
& -\sum_{a=1}^{n_{\mathrm{pts}}}\left[\sum_{e=1}^{n_{\mathrm{sd}}} \int_{\Omega^{e}} \tau_{u} v^{2} \Delta N^{b} \Delta N^{a} \mathrm{~d} \Omega\right] U_{k}^{a} \\
& +\sum_{a=1}^{n_{\mathrm{pts}}}\left[\sum_{e=1}^{n_{\mathrm{el}}} \int_{\Omega^{e}} \tau_{u} v \Delta N^{b} \partial_{k} N^{a} \mathrm{~d} \Omega\right] P^{a}
\end{aligned}
$$

The goal now is to make the convenient approximations to write this in terms of the integrals in Equations (32)-(33). First of all, observe that the integrals involving the shape functions of nodes $a$ and $b$ can be extended over $\Omega_{a} \cap \Omega_{b}$ only, the intersection of the interior of the supports of $N^{a}$ and $N^{b}$. Therefore, if approximation (40) is to be used, the functions taken out of the integrals have to be evaluated either at node $a$ or at node $b$, or a combination of both. However, it is not a matter of choice. The only possibility to approximate (55) is to take

- The velocity $\boldsymbol{a}_{h}$ appearing in the element residual of the differential equation evaluated at node $a$, and thus the $i$ th component equal to the nodal value $A_{i}^{a}$. This corresponds to the second approximation of the convective term discussed in the previous section, i.e. Equation (35) with $c=a$. The reasons for this choice are still valid in this case, whereas the argument for taking $c=b$ is not valid any more, since now, for example, $\tau_{u}\left(a_{h, i} \partial_{i} N^{b}\right)\left(\boldsymbol{x}^{a}\right)$ is not zero when $b \neq a$ (see the derivation of Equation (36)).

- The velocity $\boldsymbol{a}_{h}$ appearing in the operator applied to the test function evaluated at node $b$, and thus the $i$ th component equal to the nodal value $A_{i}^{b}$. This is needed for consistency reasons, similar to those given in Remark 6: if $\boldsymbol{a}_{h}$ depends also on node $a$, exact nodal values of the velocity would not satisfy the discrete variational equations. However, the same discussion concerning the choice of the stabilization parameters is applicable now: the resulting numerical scheme will not be conservative.

- The viscosity evaluated at node $b$. This is also due to the consistency requirement discussed in the previous section.

- The parameters $\tau_{u}$ and $\tau_{p}$ evaluated at node $b$. Even though this produces a non-conservation scheme, it is essential to have a consistent numerical method, in the sense that exact solutions of the continuous problem should also be solutions of the discrete one, provided they belong to the finite element space. The expressions we use for these parameters are (29) and (30) (and obviously (31) when the heat equation is dealt with) taking $\boldsymbol{u}_{h}$ as the nodal velocity at node $b, v$ the viscosity at this node and $h$ as the minimum distance form node $b$ to the surrounding nodes.

Using all these approximations in (55) one finds

$$
\begin{aligned}
& S_{\mathrm{mom}, 1}\left(N^{b} \boldsymbol{e}_{k} ; \boldsymbol{u}_{h}, p_{h}\right) \\
& \approx \sum_{a=1}^{n_{\mathrm{pts}}}\left[\sum_{i, j=1}^{n_{\mathrm{sd}}} A_{i}^{b} A_{j}^{a} \tau_{u}^{b}\left(\int_{\Omega} \partial_{i} N^{b} \partial_{j} N^{a} \mathrm{~d} \Omega\right)\right] U_{k}^{a}-\sum_{a=1}^{n_{\mathrm{pts}}}\left[\sum_{i=1}^{n_{\mathrm{sd}}} A_{i}^{b} \tau_{u}^{b} v^{b}\left(\sum_{e=1}^{n_{\mathrm{el}}} \int_{\Omega^{e}} \partial_{i} N^{b} \partial_{j} \Delta N^{a} \mathrm{~d} \Omega\right)\right] U_{k}^{a}
\end{aligned}
$$




$$
\begin{aligned}
& +\sum_{a=1}^{n_{\mathrm{pts}}}\left[\sum_{i=1}^{n_{\mathrm{sd}}} A_{i}^{b} \tau_{u}^{b}\left(\int_{\Omega} \partial_{i} N^{b} \partial_{k} N^{a} \mathrm{~d} \Omega\right)\right] P^{a}+\sum_{a=1}^{n_{\mathrm{pts}}}\left[\sum_{j=1}^{n_{\mathrm{sd}}} A_{j}^{a} v^{b} \tau_{u}^{b}\left(\sum_{e=1}^{n_{\mathrm{el}}} \int_{\Omega^{e}} \Delta N^{b} \partial_{j} N^{a} \mathrm{~d} \Omega\right)\right] U_{k}^{a} \\
& -\sum_{a=1}^{n_{\mathrm{pts}}}\left[\left(v^{b}\right)^{2} \tau_{u}^{b}\left(\sum_{e=1}^{n_{\mathrm{el}}} \int_{\Omega^{e}} \Delta N^{b} \Delta N^{a} \mathrm{~d} \Omega\right)\right] U_{k}^{a}+\sum_{a=1}^{n_{\mathrm{pts}}}\left[v^{b} \tau_{u}^{b}\left(\sum_{e=1}^{n_{\mathrm{el}}} \int_{\Omega^{e}} \Delta N^{b} \partial_{k} N^{a} \mathrm{~d} \Omega\right)\right] P^{a}
\end{aligned}
$$

Once again, the objective of using only the integrals in (32)-(33) has been accomplished.

\section{Remark 7}

It is interesting to note that even when $\boldsymbol{a}_{h}=0$, the final algebraic system of equations will not be symmetric if the stabilization parameters change from node to node. That is, if the mesh is not uniform or the viscosity is variable.

The way to treat the stabilization terms (48) and (49), and also the one coming from the stabilization of the heat equation when this is solved, is exactly the same as for (47): the stabilization parameters must be evaluated at the node associated to the test function being used and the convective velocity and viscosity evaluated as explained above.

\section{NUMERICAL EXAMPLES}

In this section we present the results of some numerical examples obtained with the stabilized finite element formulation proposed in this paper. Two types of conclusions can be drawn from them. On the one hand, these examples serve to check the behaviour of the stabilized formulation in situations more general than those in which it can be analysed [3]. These situations include thermally driven flows, flows of non-linear materials and transient problems. An example of each situation is presented.

On the other hand, these numerical examples also serve to compare the performance of the standard element-based implementation of the stabilized formulation and the nodal-based one presented in Section 4, which involves several additional approximations.

The last two examples are intended to demonstrate that the work developed in Section 4 'makes sense'. Firstly, because the resulting numerical scheme is shown to be (almost) optimally convergent in a numerical test, despite all the approximation needed to arrive to it, and secondly because this scheme turns out to be very efficient, an attribute of which finite element methods are usually blamed for lacking.

\subsection{Thermally coupled flow in a cavity}

In this example, the convective motion of a fluid enclosed in the square cavity $[0,1] \times[0,1]$ and driven by a temperature gradient will be numerically analysed. The left vertical wall $x=0$ is heated and maintained at a constant temperature $\vartheta=1$, while the right vertical wall $x=1$ is kept at $\vartheta=0$. Horizontal walls are assumed to be adiabatic, i.e. boundary condition (9) with $\bar{h}=0$ is prescribed. Homogeneous Dirichlet boundary conditions are prescribed everywhere on the boundary for the velocity. 
Let $L$ be a characteristic length of the problem and $G_{\vartheta}$ a characteristic temperature gradient. The Grashof number $\mathrm{Gr}$ and the Prandtl number $\mathrm{Pr}$ are defined as

$$
G r:=\frac{\beta|\boldsymbol{g}| L^{3} G_{\vartheta}}{v^{2}}, \quad \operatorname{Pr}:=\frac{v}{\kappa}
$$

Taking $L=1$ and $G_{\vartheta}=1$ in this case, the physical parameters have been adjusted to yield a Prandtl number $\operatorname{Pr}=0.005$ and a Grashof number $G r=3 \times 10^{6}$. For this combination of values, there is a unique and stable stationary solution to the Navier-Stokes equations coupled with the heat equation using the Boussinesq assumption [16]. Thus, the stationary version of problem (1)-(3) is solved in this example. The gravity is assumed to point downwards.

The finite element mesh employed to discretize the problem, which is refined near the boundaries, consists of 2684 bilinear $\left(Q_{1}\right)$ elements and 2809 nodal points. The Navier-Stokes equations have been linearized up to first order, and the standard penalty method, with $\epsilon=10^{-6}$, has been used to fix the pressure mean to zero. The convergence tolerance has been taken as 0.01 per cent in the relative $L^{2}$ norm.

The velocity field is shown in Figure 1 . These results are very similar using the standard element-based implementation and the nodal-based one presented in this paper. As a sample, a comparison is made in Figure 2, where the $y$-velocity section $x=0.5$ is shown. It is important to remark that this example involves two of the approximations discussed in Section 4, namely those related to the convective term for both the Navier-Stokes and the heat transport equations, and the approximations needed to deal with their stabilization terms.

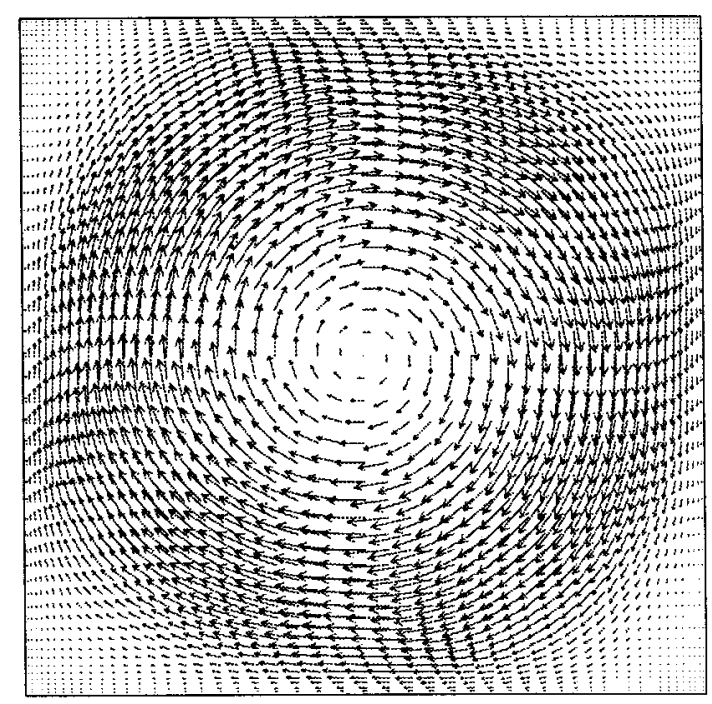

Figure 1. Velocity vectors for Example 1. 


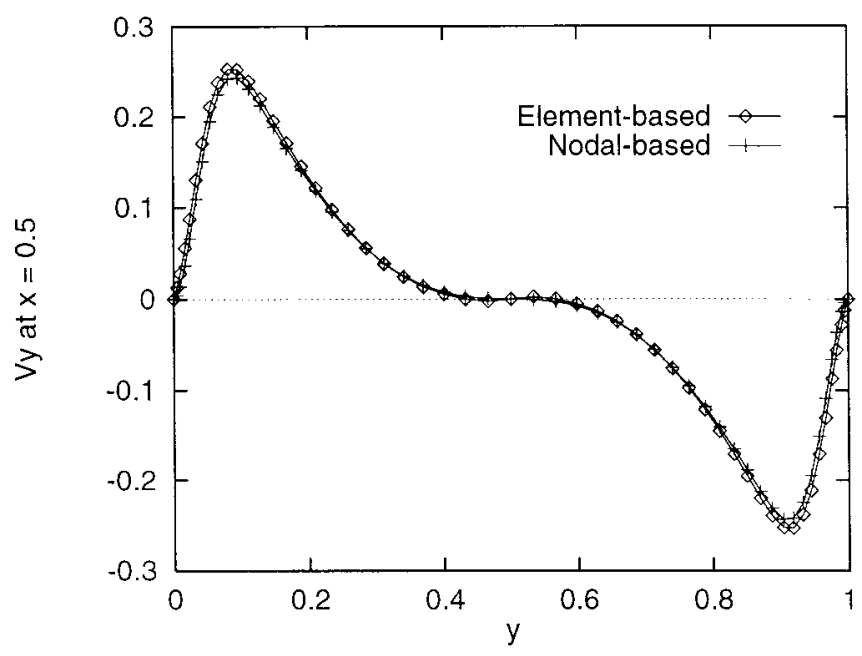

Figure 2. $y$-Velocity at $x=0.5$ for Example 1 .

\subsection{Flow over a cylinder}

This example involves the flow past a cylinder, another widely solved benchmark problem. The computational domain is $\bar{\Omega}=[0,16] \times[0,8] \backslash D$, with the cylinder $D$ of diameter 1 and centred at $(4,4)$. The velocity at $x=0$ is prescribed at $(1,0)$, whereas at $y=0$ and $y=8$, the $y$-velocity component is prescribed at 0 and the $x$-component is left free. The outflow (where both the $x$ and $y$-components are free) is $x=16$. The Reynolds number is 100 , based on the cylinder and the prescribed inflow velocity. The finite element mesh employed consists of 4000 linear triangles, with 2100 nodal points, and is refined near the cylinder.

In order to obtain the fully developed vortex shedding characteristic of this problem, 90 times steps have been performed with $\delta t=1$ and $\alpha=0.5$ (Crank-Nicholson scheme), employing for that the element-based implementation. The convergence tolerance within each time step has been taken as 1 per cent (a single Picard iteration has been needed to converge). The solution thus obtained shows a fully developed periodic flow pattern. These results have been taken as the initial condition for a more accurate calculation, now computed with $\delta t=0.1$ and requiring a convergence tolerance of 0.01 per cent in the relative $L_{2}$ norm. Two or three Newton-Raphson iterations have been performed for each time step, both for the element based and the nodal based implementations of the formulation.

The period of the oscillations has been found to be 5.9 times units with both implementations. The values given in References [17,18] are 6.0 and 5.6 respectively. In Reference [19], the period obtained with a very fine mesh (3426 $Q_{2} / P_{1}$ elements, 14000 nodal points) is 5.8 time units. See also Reference [20] for results obtained using a similar stabilized formulation.

The streamline snapshot at $t=10$ is shown in Figure $3(t=0$ corresponds to the periodic solution computed as described earlier with a higher tolerance and a higher time step size). 


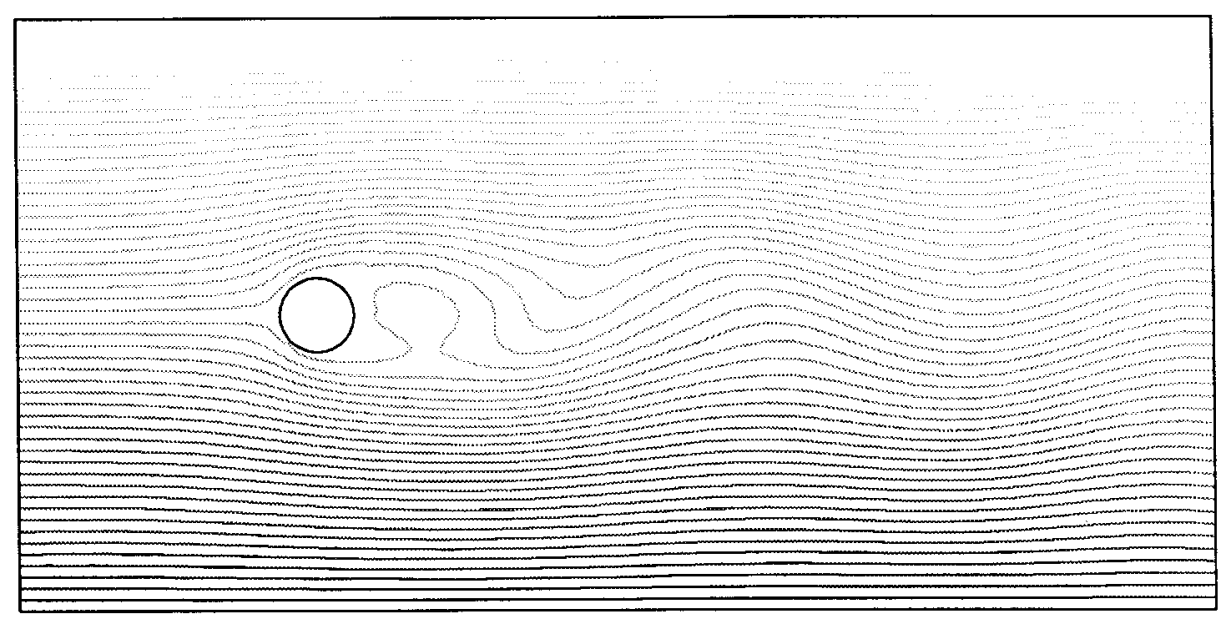

Figure 3. Streamlines at $t=10$ for Example 2.

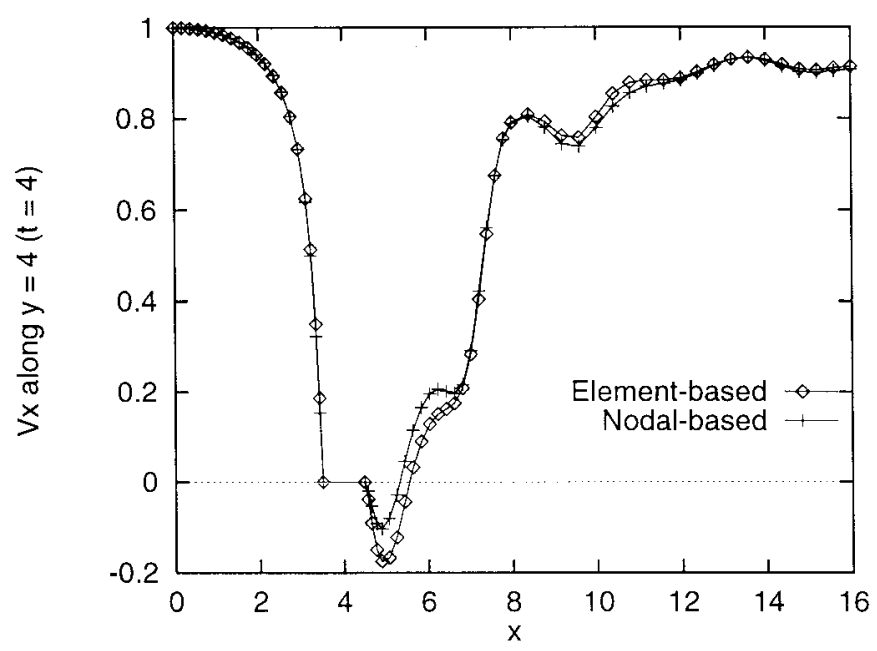

Figure 4. $x$-Velocity at $y=4$ and $t=4$ for Example 2 .

The important point is the comparison of the results obtained with the element-based and the nodal-based implementations. An example of this comparison is shown in Figures 4 and 5. It is observed that there are only slight differences in the spatial amplitude of the oscillations. 


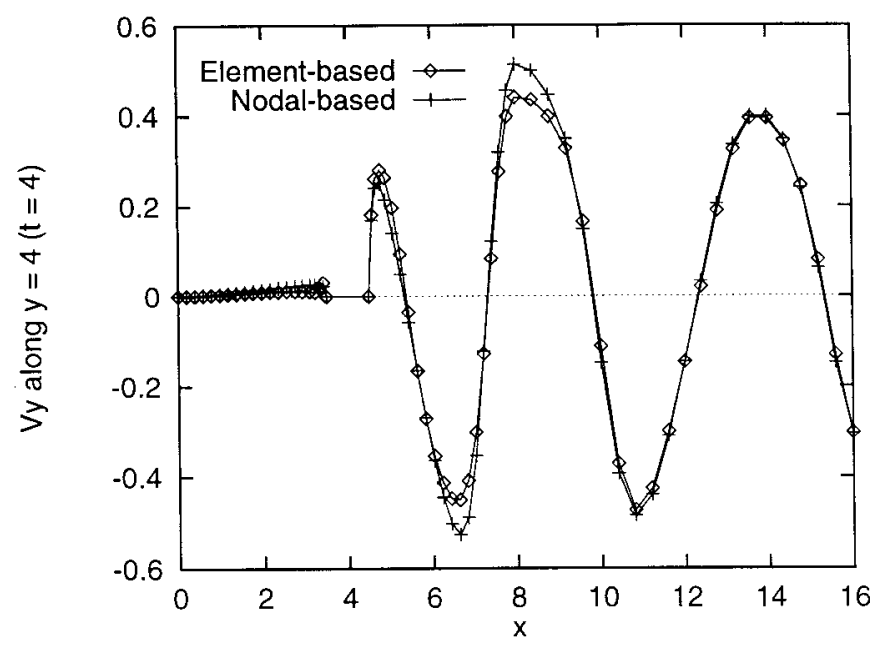

Figure 5. $y$-Velocity at $y=4$ and $t=4$ for Example 2 .

\subsection{Extrusion of a non-linear fluid}

In this section we present some numerical results obtained for the well-known 4:1 plane extrusion problem. This is a very popular test for non-Newtonian flows, since all the flow features that characterize these fluids are present in this problem.

The computational domain in this case is $[0,20] \times[0,4] \cup[20,40] \times[3,4]$. The inflow is the section $x=0$, where the velocity field is prescribed to $\left(v_{x}(y), 0\right)$, where $v_{x}(y)$ has a parabolic profile with maximum 1 at $y=4$ and minimum 0 at $y=0$. The outflow $x=40$ is left free, i.e. condition (7) with $\overline{\boldsymbol{t}}=\mathbf{0}$ is prescribed. On $y=4$ the velocity is set to $(1,0)$ and on the rest of the boundary the non-slip condition is used.

The finite element mesh employed for the space discretization is composed of 2100 bilinear $\left(Q_{1}\right)$ elements, with a total of 2201 nodal points. There are 15 elements in the $y$-direction from co-ordinates $y=3$ to $y=4$ and only 12 from $y=0$ to $y=3$. The concentration of elements in the former zone is needed if one wants to reproduce accurately the shear thinning effect of fluids whose viscosity obeys the power law that we consider now, given by (5). Since the effective viscosity values that result from this law are very high, the convective term of the Navier-Stokes equations is neglected (the flow is assumed to be governed by creep), as well as the buoyancy forces due to temperature (the flow is assumed to be thermally uncoupled).

The values of the physical constants that have been used are (all in SI units): $\rho_{0}=1200$ (density), $K_{0}=10^{6}$ (material consistency) and $n=0.4$ (rate sensitivity). For this value of $n$ the effect of the non-constant viscosity is pronounced. Since the expressions of the viscosity (5) tends to infinity when $I^{2}(\varepsilon)$ tends to zero, a cut-off value $v_{\mathrm{c}}=10^{12}$ for $v$ has been introduced. The values of the viscosity for the converged solutions are always below this limit, except in isolated points.

The streamlines for this problem are shown in Figure 6. As before, results are very similar using the element-based and the nodal-based implementations. A comparison is made in 


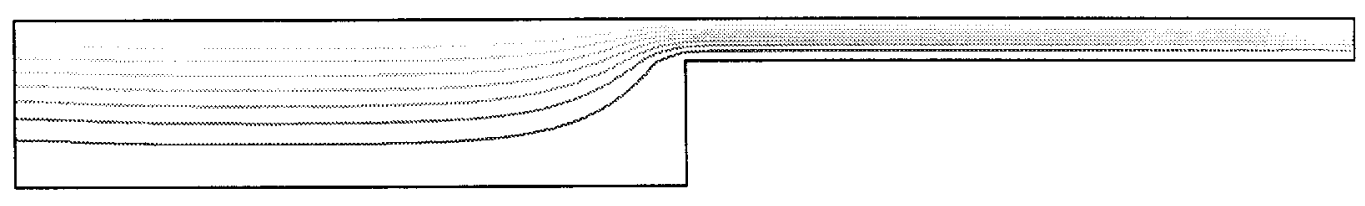

Figure 6. Streamlines for Example 3.

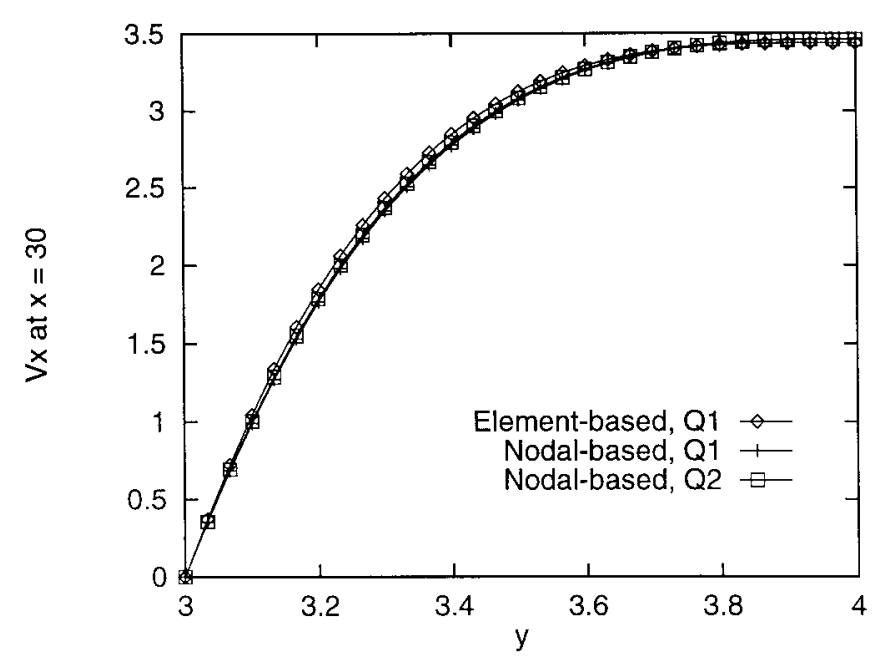

Figure 7. $x$-Velocity at $x=30$ for Example 3 .

Figures 7 and 8, where the $x$ - and $y$-velocity components at $x=30$ and $y=3.5$ are shown. In these figures the results obtained using a mesh of 525 biquadratic $\left(Q_{2}\right)$ elements with the same set of nodes as the mesh of bilinear elements are also shown for the nodal-based implementation. It has to be remarked that the element based one did not converge using $Q_{2}$ elements.

\subsection{Convergence test}

Let us consider now a two-dimensional steady state test with analytical solution to check the behaviour in space of the finite element approximation to problem (1)-(3). We take $\Omega$ as the unit square and the force term so that the exact solution is $p=0$ and $\boldsymbol{u}(x, y)=\left(f(x) g^{\prime}(y)\right.$, $\left.-f^{\prime}(x) g(y)\right)$, with $f(x)=x^{2}(1-x)^{2}$ and $g(y)=y^{2}(1-y)^{2}$. This velocity field vanishes on $\partial \Omega$.

As physical properties we have taken $v=0.001$ and different values of $\omega=|\omega|$ and $\sigma$. In particular, results will be shown for $\sigma=0,1000$ and $\omega=0,1000$. We have used three uniform finite element meshes (meshes 1, 2 and 3) of $5 \times 5,10 \times 10$ and $20 \times 20$ biquadratic elements, so that the element sizes are $h=0.2, h=0.1$ and $h=0.05$ respectively. The resulting values of the element Reynolds number are not very high and for this particular example the standard 


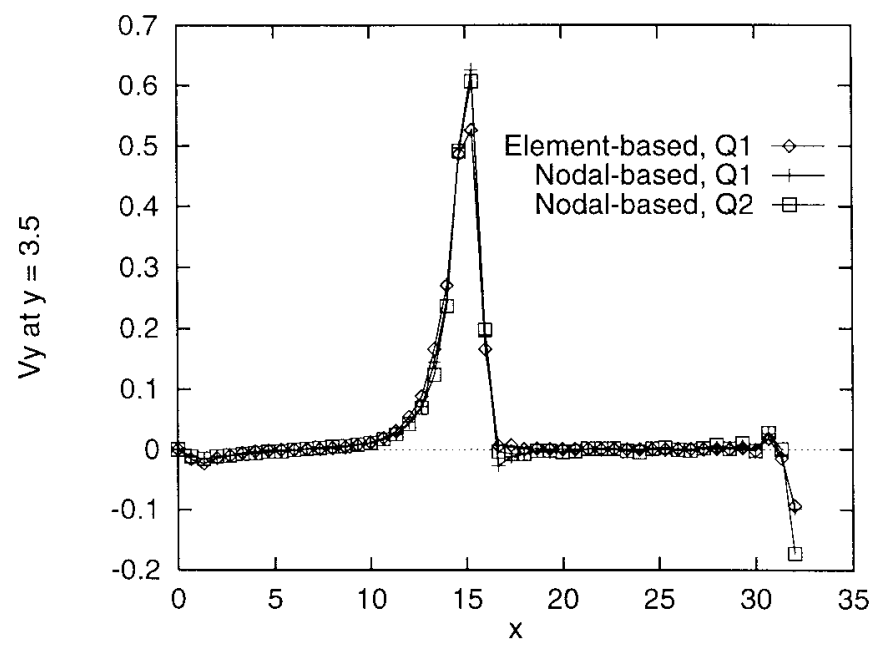

Figure 8. $y$-Velocity at $y=3.5$ for Example 3.

Galerkin approach using a stable velocity-pressure pair, such as the Taylor-Hood element $Q_{2} / Q_{1}$, works for meshes 2 and 3 in the absence of Coriolis force. However, when this force exists, the Galerkin method yields completely oscillatory results all over the computational domain (see Reference [3]).

In Figure 9 we have plotted the convergence of the velocities obtained with the stabilized method as the mesh is refined in the $\ell^{2}$ norm and for different combinations of the values of $\sigma$ and $\omega$. This error is defined as

$$
E=\left[\sum_{a=1}^{n_{\mathrm{pts}}} \sum_{i=1}^{n_{\mathrm{sd}}}\left(U_{i}^{a}-u_{i}\left(\boldsymbol{x}^{a}\right)\right)^{2}\right]^{1 / 2}\left[\sum_{a=1}^{n_{\mathrm{pts}}} \sum_{i=1}^{n_{\mathrm{sd}}}\left(u_{i}\left(\boldsymbol{x}^{a}\right)\right)^{2}\right]^{-1 / 2}
$$

where $\boldsymbol{x}^{a}$ are the co-ordinate of the nodes.

The optimal convergence rate that should be expected is 3 . From Figure 9 it is seen that this is what is found for the element-based implementation. However, for the nodal-based one it is slightly smaller when $\sigma=\omega=0$ (it is approximately 2.6). This is due to the approximation made for the convective term, which is the dominant one in this case.

A comparison of results for $c=a$ and for $c=b$ in (35) (and the corresponding approximation for the convective part of the stabilization term) is shown in Figure 10. It is seen there that the choice $c=a$ (employed also in Figure 9) gives better results than $c=b$. The convergence rate obtained using a mesh of $Q_{1}$ elements obtained by splitting each $Q_{2}$ element into four is also plotted in Figure 10 (using again $c=a$ ). In this case, convergence is optimal, with rate 2 . 


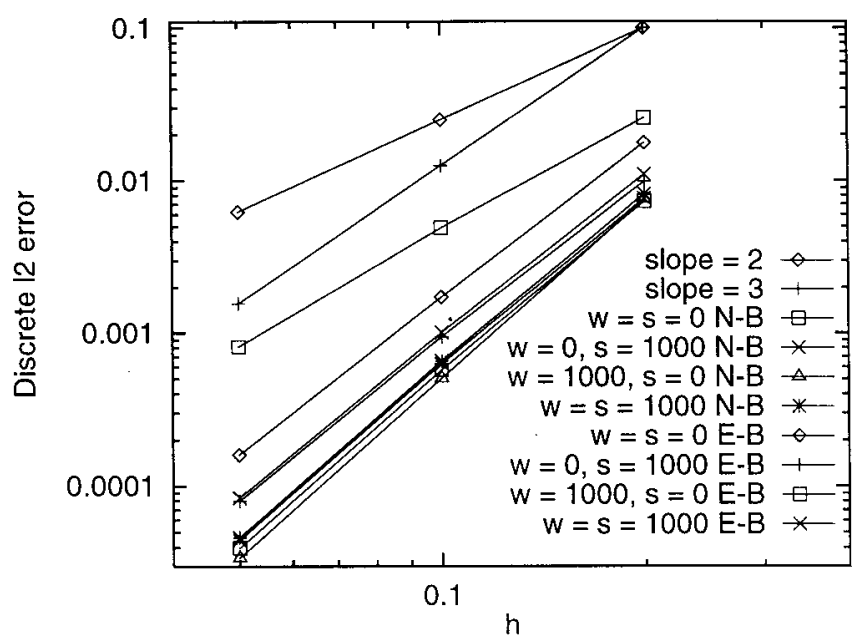

Figure 9. Discrete $\ell^{2}$ errors for Example 4 for different values of $|\omega| \equiv \omega$ and $\sigma \equiv s$ for the element-based (E-B) and nodal based (N-B) implementations.

\subsection{Efficiency test}

In this final example, the efficiency of the nodal-based implementation compared with the element-based one is studied. For that, we considered the three-dimensional extension of the previous example. The domain is the unit cube $\bar{\Omega}=[0,1] \times[0,1] \times[0,1]$ and is discretized using three meshes of $P_{1}, P_{2}, Q_{1}$ and $Q_{2}$ elements.

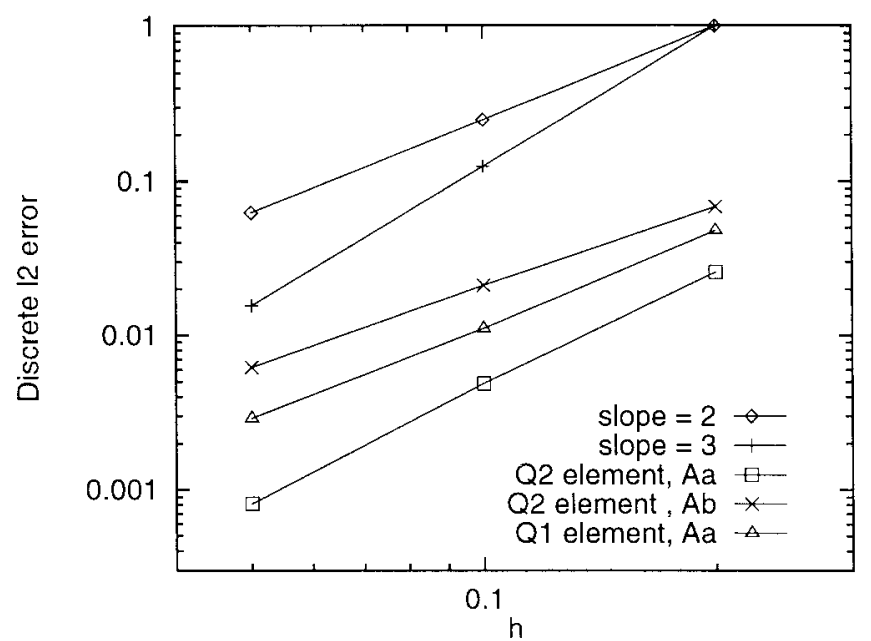

Figure 10. Discrete $\ell^{2}$ errors for Example 4 for $Q_{2}$ and $Q_{1}$ elements using $A^{a}$ or $A^{b}$ in (35). 
The interest of this example is to compare the CPU time needed to construct the stiffness matrix of the final linear system for the two implementations of the stabilized method. Although this linear system has not been solved, the force term has been taken so as to obtain as exact solution $\boldsymbol{u}(x, y, z)=\left(h(z) f(x) g^{\prime}(y),-h(z) f^{\prime}(x) g(y), 0\right)$, with $f(x)$ and $g(y)$ as in the previous example and $h(z)=z(1-z)$. Likewise, $\omega=(1,1,1)$ and $\sigma=1000$ have been used.

Results are shown in Table I where the meshes employed have been identified as follows: ' $t$ ' corresponds to tetrahedral elements and ' $h$ ' to hexahedral, ' $l$ ' to linear and ' $q$ ' to quadratic interpolations, and ' 1 ', ' 2 ', ' 3 ' identify the number of nodes of the mesh. These meshes have respectively $7^{3}, 13^{3}$ and $25^{3}$ nodal points, equally distributed.

For the case of tetrahedral linear elements $\left(P_{1}\right)$, i.e. for meshes $\mathrm{tl}$, t12 and $\mathrm{t} 13$, two possibilities have been considered, namely, the use of four points to perform the numerical integration (which corresponds to 'full' integration) and one point (which introduces an important numerical integration error but is possible for this stationary problem). Obviously, this does not affect the CPU time needed for the construction of the stiffness matrix in the nodal-based implementation, since integrals are computed and stored at the beginning of the calculations. Similarly, for tetrahedral quadratic elements $\left(P_{2}\right)$, both 11 points and four points can be used for the numerical integration.

The results shown in Table I, obtained using a single SGI R10000 processor, show that the nodal-based implementation is clearly much more efficient than the element-based one. Obviously these results are dependent on the particular coding of the corresponding finite element algorithm, but this can not affect the general tendency observed in Table I.

Let us consider now the memory storage required for both implementations. If the number of nodal points is increased, the asymptotic number of resulting elements is $n_{\mathrm{el}}=f_{\mathrm{ele}} n_{\mathrm{pts}}$, where

Table I. Comparison of the CPU time needed to construct the stiffness matrix in Example 5.

\begin{tabular}{lccc}
\hline Mesh & Element-based & Nodal-based & Factor \\
\hline t11, four points & 0.94 & 0.06 & 15.67 \\
t11, one point & 0.35 & 0.06 & 5.83 \\
h11 & 0.71 & 0.09 & 7.89 \\
tq1, 11 points & 0.87 & 0.10 & 8.70 \\
tq1, four points & 0.43 & 0.10 & 4.30 \\
hq1 & 2.48 & 0.21 & 11.81 \\
t12, four points & 7.57 & 0.44 & 17.20 \\
t12, one point & 2.81 & 0.44 & 6.39 \\
h12 & 5.67 & 0.68 & 8.34 \\
tq2, 11 points & 6.89 & 0.79 & 8.72 \\
tq2, four points & 3.44 & 0.79 & 4.35 \\
hq2 & 19.98 & 1.87 & 10.64 \\
t13, four points & 60.52 & 3.87 & 15.64 \\
t13, one point & 22.65 & 3.87 & 5.85 \\
h13 & 44.60 & 6.14 & 7.26 \\
tq3, 11 points & 55.19 & 7.10 & 7.77 \\
tq3, four points & 27.14 & 7.10 & 3.82 \\
hq3 & 165.04 & 15.95 & 10.35 \\
\hline
\end{tabular}


Table II. Comparison of the asymptotic number of coefficients needed to store derivatives for the element-based implementation and integrals for the nodalbased implementation.

\begin{tabular}{lllll}
\hline Mesh & $f_{\text {ele }}$ & $\mathscr{M}_{\text {eb }}$ & $f_{\text {con }}$ & $\mathscr{M}_{\text {nb }}$ \\
\hline tl, four points & 6 & 336 & 9 & 58.5 \\
tl, one point & 6 & 102 & 9 & 58.5 \\
hl & 1 & 208 & 27 & 175.5 \\
tq, 11 points & 0.75 & 263.25 & 26.625 & 173.0625 \\
tq, four points & 0.75 & 100.5 & 26.625 & 173.0625 \\
hq & 0.125 & 280.125 & 64 & 416 \\
\hline
\end{tabular}

$f_{\text {ele }}$ is a factor that depends on the element type. Similarly, if $f_{\text {con }}$ is the average number of nodes to which a node is connected, the number of non-zero coefficients of the mesh graph is $\mathrm{NZD}=f_{\text {con }} n_{\mathrm{pts}}$. Let $\mathscr{M}_{\mathrm{eb}} n_{\mathrm{pts}}$ be the number of coefficient that need to be stored to compute the stiffness matrix for the element based implementation and $\mathscr{M}_{\mathrm{nb}} n_{\mathrm{pts}}$ for the nodal based one. Assuming in both cases that no second derivatives need to be computed the coefficients $\mathscr{M}_{\mathrm{eb}}$ and $\mathscr{M}_{\mathrm{nb}}$ are

$$
\begin{aligned}
\mathscr{M}_{\mathrm{eb}}= & {\left[(1 \text { (element of volume })+n_{\mathrm{sd}} n_{\text {nod }}(\text { Cartesian derivatives })\right) n_{\mathrm{int}} } \\
& \left.+n_{\text {nod }}(\text { nodal convectivities })\right] f_{\text {ele }} \\
\mathscr{M}_{\mathrm{nb}}= & 0.5(1 \text { (first integrals in (32) })+n_{\mathrm{sd}}(\text { second integrals in }(32)) \\
& \left.+n_{\mathrm{sd}} n_{\mathrm{sd}}(\text { last integrals in }(32))\right) f_{\text {con }}
\end{aligned}
$$

Observe that in the expression $\mathscr{M}_{\mathrm{nb}}$ we have taken into account the symmetries of the integrals in (32) as well as the fact that

$$
\int_{\partial \Omega} n_{i} N^{a} N^{b} \mathrm{~d} \Gamma=\int_{\Omega} \partial_{i} N^{a} N^{b} \mathrm{~d} \Omega+\int_{\Omega} N^{a} \partial_{i} N^{b} \mathrm{~d} \Omega, \quad i=1, \ldots, n_{\mathrm{sd}}
$$

which allows us to store only one of the integrals in the second row of (32). Likewise, for the element-based implementation the storage of the nodal convectivities has to be accounted for whereas for the nodal-based one the mesh graph can be considered as part of the memory needed to allocate the stiffness matrix of the problem.

The coefficients $\mathscr{M}_{\mathrm{eb}}$ and $\mathscr{M}_{\mathrm{nb}}$ for the different types of meshes in terms of the factors $f_{\text {ele }}$ and $f_{\text {con }}$ are given in Table II. It is observed there that the nodal-based implementation is less memory demanding for lower-order interpolations and for fully integrated quadratic tetrahedra, whereas the memory required is more than for the element-based implementation for quadratic hexahedra.

\section{CONCLUSIONS}

Two different aspects related to the finite element approximation of the incompressible Navier-Stokes equations have been treated in this paper. The first of them is the numerical 
formulation, which consists of a stabilized method able to deal with a very wide range of flows. It has been shown that the method gives very good results for thermally driven flows, non-Newtonian fluids and transient problems. Particular features of this stabilized formulation are that it is based on multiplying the element residuals of the equations by the adjoint of the linearized Navier-Stokes operator applied to the test functions, and also the design of the stabilization parameters. Both ingredients allow to stabilize very different types of numerical instabilities, namely those that are classical and arise in convection dominated flows and equal velocity-pressure interpolations, and also the less studied cases of dominating Coriolis forces and small medium permeabilities.

The good numerical performance of the stabilized finite element formulation is maintained when the nodal-based implementation described here is used. The basic idea of this implementation is to characterize the topology of the finite element mesh by the matrix of its graph. The arrays needed for it can be used also to store the integrals of the shape functions and shape function derivative products, the corner stones of the implementations. All the arrays appearing in the fully discrete problem can be expressed in terms of them after performing the appropriate approximations. Numerical experiments show that the good numerical results due to the stabilized formulation can be obtained in an efficient manner by means of this implementation.

\section{REFERENCES}

1. Hughes TJR. Multiscale phenomena: Green's function, the Dirichlet-to-Neumann formulation, subgrid scale models, bubbles and the origins of stabilized formulations. Computer Methods in Applied Mechanics and Engineering 1995; 127: 387-401.

2. Codina R. On stabilized finite element methods for linear systems of convection-diffusion-reaction equations. Computer Methods in Applied Mechanics and Engineering 2000 (to appear).

3. Codina R. A stabilized finite element method for generalized stationary incompressible flows. Computer Methods in Applied Mechanics and Engineering 2000 (to appear).

4. Brezzi F, Fortin M. Mixed and Hybrid Finite Element Methods. Springer: Berlin, 1991.

5. Hughes TJR, Franca LP, Balestra M. A new finite element formulation for computational fluid dynamics: V. Circumventing the Babuška-Brezzi condition: a stable Petrov-Galerkin formulation for the Stokes problem accommodating equal-order interpolations. Computer Methods in Applied Mechanics and Engineering 1996; 59: 85-99.

6. Franca LP, Frey SL. Stabilized finite element methods: II. The incompressible Navier-Stokes equations. Computer Methods in Applied Mechanics and Engineering 1992; 99: 209-233.

7. Hansbo P, Szepessy A. A velocity-pressure streamline diffusion finite element method for the incompressible Navier-Stokes equations. Computer Methods in Applied Mechanics and Engineering 1990; 84: 175-192.

8. Barth T. A 3D upwind Euler solver for unstructured meshes. AIAA-91-1548, 1991.

9. Mavriplis D. Three-dimensional unstructured multigrid for the Euler equations. AIAA-91-1549, 1991.

10. Luo H, Baum JD, Löhner R, Cabello J. Adaptive edge-based finite element schemes for the Euler and Navier-Stokes equations on unstructured grids. AIAA 93-0336, 1993.

11. Heywood JG, Rannacher R. Finite element approximation of the nonstationary Navier-Stokes problem. IV: error analysis for second-order time discretization. SIAM Journal on Numerical Analysis 1990; 27: 353-384.

12. Lube G, Weiss D. Stabilized finite element methods for singularly perturbed parabolic problems. Applied Numerical Mathematics 1995; 17: 431-459.

13. Cervea M, Codina R, Galindo M. On the computational efficiency and implementation of block-iterative algorithms for nonlinear coupled problems. Engineering Computations 1996; 13(6): 4-30.

14. Codina R. Comparison of some finite element methods for solving the diffusion-convection-reaction equation. Computer Methods in Applied Mechanics and Engineering 1998; 156: 185-210.

15. Codina R. An iterative penalty method for the finite element solution of the stationary Navier-Stokes equations. Computer Methods in Applied Mechanics and Engineering 1993; 110: 237-262. 
16. Mohamad AA, Viskanta R. Transient natural convection of low-Prandtl number fluids in a differentially heated cavity. International Journal of Numerical Methods in Fluids 1991; 13: 61-81.

17. Brooks AN, Hughes TJR. Streamline upwind/Petrov-Galerkin formulations for convection dominated flows with particular emphasis on the incompressible Navier Stokes equation. Computer Methods in Applied Mechanics and Engineering 1982; 32: 199-259.

18. Gresho PM, Chan ST, Lee RL, Upson CD. A modified finite element method for solving the time-dependent incompressible Navier-Stokes equations. Part 2: applications. International Journal for Numerical Methods in Fluids 1984; 4: 619-640.

19. Engelman MS, Jamnia MA. Transient flow past a circular cylinder: a benchmark solution. International Journal for Numerical Methods in Fluids 1990; 11: 985-1000.

20. Tezduyer TE, Mittal S, Ray SE, Shih R. Incompressible flow computations with stabilised bilinear and linear equal-order interpolation velocity-pressure elements. Computer Methods in Applied Mechanics and Engineering 1992; 95: 221-242. 